1 Mesenchymal tumor organoid models recapitulate

\title{
2 rhabdomyosarcoma subtypes
}

3

4 Michael T. Meister ${ }^{1}$, Marian J. A. Groot Koerkamp ${ }^{1}$, Terezinha de Souza ${ }^{1}$, Willemijn

${ }^{1}$ Princess Máxima Center for Pediatric Oncology, Heidelberglaan 25, 3584CS Utrecht, The Netherlands.

2 Department of Oncology and Children's Research Center, University Children's Hospital Zürich, 8032 Zürich, Switzerland.

${ }^{3}$ Oncode Institute, Heidelberglaan 25, 3584CS Utrecht, The Netherlands.

${ }^{4}$ Children's Cancer Institute, Lowy Cancer Centre, UNSW Sydney, Kensington, NSW, Australia.

${ }^{5}$ School of Women's and Children's Health, Faculty of Medicine, UNSW Sydney, Kensington, NSW, Australia.

${ }^{6}$ Center for Molecular Medicine, UMC Utrecht and Utrecht University, Utrecht, The Netherlands.

*Correspondence: f.c.p.holstege@prinsesmaximacentrum.nl 


\section{Summary}

27 Rhabdomyosarcomas (RMS) are mesenchyme-derived tumors and the most common

28 childhood soft tissue sarcomas. Treatment is intense, with a nevertheless poor

29 prognosis for high-risk patients. Discovery of new therapies would benefit from

30 additional preclinical models. Here we describe the generation of a collection of pediatric RMS tumor organoid (tumoroid) models comprising all major subtypes. For aggressive tumors, tumoroid models can often be established within four to eight weeks, indicating the feasibility of personalized drug screening. Molecular, genetic and histological characterization show that the models closely resemble the original tumors, with genetic stability over extended culture periods of up to six months. Importantly, drug screening reflects established sensitivities and the models can be modified by CRISPR/Cas9 with TP53 knockout in an embryonal RMS model resulting in replicative stress drug sensitivity. Tumors of mesenchymal origin can therefore be used to generate organoid models, relevant for a variety of preclinical and clinical research questions.

Keywords: rhabdomyosarcoma, mesenchymal, tumor organoid, pediatric cancer, drug screening, CRISPR/Cas9

Running title: A mesenchymal tumor organoid collection

Tables: 2 supplementary

Figures: 6 main and 5 supplementary 


\section{Introduction}

51 Rhabdomyosarcoma (RMS) is a type of malignant tumor of mesenchymal origin (Yang et al., 2014) and forms the most common soft tissue sarcoma in children and adolescents ( $\mathrm{Li}$ et al., 2008). Historically, RMS has been divided into two main subtypes based on histology. Whereas embryonal RMS (eRMS) displays cellular heterogeneity and hallmarks of immature skeletal myoblasts (Patton and Horn, 1962), alveolar RMS (aRMS) cells are distributed around an open central space, thereby resembling pulmonary alveoli (Enterline and Horn, 1958). eRMS is more frequently observed in children under ten, accounting for two-thirds of all RMS cases, and generally has a better prognosis than aRMS, which is more common in adolescents and young adults (Perez et al., 2011). In aRMS, a sole genetic driver alteration is usually observed, caused by a chromosomal translocation resulting in a fusion gene between either $P A X 3$ or $P A X 7$ and FOXO1. In contrast, eRMS is genetically more heterogeneous, harboring mutations in several common oncogenes or tumor suppressor genes (Shern et al., 2014). Other subtypes of RMS have recently been recognized (WHO, 2020). RMS treatment is guided by protocols developed by multinational collaborative groups and includes systemic chemotherapy in addition to local therapy (radiotherapy and/or surgery) (Skapek et al., 2019). The prognosis of RMS has improved over the last decades (Bisogno et al., 2019). For patients with high-risk, refractory or relapsed disease, prognosis remains poor however, despite an immense treatment burden (Mascarenhas et al., 2019; Pappo et al., 1999). Thus, development of new therapeutic options is of critical importance for these patients.

Development of such treatment options requires in vitro models and may therefore benefit from application of organoid technology. The basis of this technology is that 
given a suitable growth environment, tissue stem cells self-renew as well as give rise to natural progeny which organize according to their preferred growth modality without the need for artificial cell immortalization. The technology was first established in healthy epithelial tissue from mouse small intestine (Sato et al., 2009) and soon adapted to various other healthy and diseased epithelial tissues, including cancer (Clevers, 2016). Tumor organoid (tumoroid) systems are proving useful in cancer research as they display genetic stability over extended culture periods, retaining the molecular characteristics of the tumor they are derived from. Tumoroid models can be expanded, facilitating high-throughput screening approaches such as small molecule or CRISPR/Cas9-knockout screening (Bleijs et al., 2019).

85

To date, tumoroid approaches have been primarily applied to cancers derived from epithelial cells (i.e., carcinomas). Recent studies demonstrate that deriving tumoroid models from non-epithelial cancer is feasible but this has as yet not been achieved for pure mesenchymal cancers (Abdullah et al., 2021; Fusco et al., 2019; Jacob et al., 2020; Saltsman et al., 2020; Yamazaki et al., 2021). Application to tumors of mesenchymal origin such as RMS would be of obvious benefit. Tumoroid models of pediatric nephroblastoma (Wilms tumors) have been described, which, depending on the subtype, can contain stromal cells (Calandrini et al., 2020). In addition, cells derived from synovial sarcoma and other adult soft tissue sarcomas can grow to a limited extent on fetal calf serum, which, although undefined in terms of the required essential growth factors, also indicates feasibility (Boulay et al., 2021; Brodin et al., 2019). Furthermore, in vitro propagation of RMS tumor cells derived from patientderived xenograft (PDX) mouse models has recently been shown (Manzella et al., 2020). Although these results are encouraging, no directly patient-derived collection 
100 of tumoroid models of malignant tumors of pure mesenchymal origin (i.e., sarcomas)

101 has been generated and studied after growth for extensive periods in well-defined

102 media components. In this study, we therefore set out to develop and apply

103 approaches for generating a collection of tumoroid models that covers the major RMS

104 subtypes, a pediatric cancer of mesenchymal origin with poor outcome for high-risk

105 patients. Besides generating and extensively characterizing the tumoroid collection,

106 we also investigated applicability for drug screening and genetic modification (Fig. 1A).

107 


\section{Results}

109 A protocol to collect and process RMS tumor samples for tumoroid model

\section{0 establishment and propagation}

111 Before starting to generate a collection of RMS tumoroid models, we first optimized

112 sample acquisition and logistics between surgery, pathology and organoid culture labs

113 (Materials \& Methods). In parallel to optimizing sample acquisition, we also optimized

114 sample processing, including testing different formulations of growth media by a

115 combination of systematic and trial and error approaches (Discussion). RMS tumor

116 samples are diverse. Most samples are small needle biopsies (i.e, 16-gauge tru-cut),

117 as large resection specimens are mostly restricted to pre-treated RMS or to treatment-

118 naïve paratesticular fusion-negative eRMS (FN-eRMS). In addition, a subset of 119 samples (4\% here) are not solid, being acquired as bone marrow aspirates of

120 infiltrating tumor cells (Fig. 1B). Samples are plated as minced pieces embedded in a

121 droplet of extracellular matrix (ECM) substitute (Basement-Membrane Extract, BME)

122 and as single-cell suspensions in BME-supplemented medium. Outgrowth of tumor

123 cells to tumoroid models can occur from both modalities. In the case of successful

124 outgrowth of initially plated cells, cells organize as two-dimensional monolayers (Fig.

125 1C). This appears to be the cells' preferred growth modality, as plating them as single-

126 cell suspensions in BME droplets results in cells escaping the surrounding matrix and

127 sinking to the bottom of the culture plate from which they continue to grow in a

128 monolayer. Therefore, cells are further propagated and expanded in this way. We

129 considered an RMS tumoroid model to be successfully established if, over the course

130 of culturing, the expression of specific tumor markers is retained and the culture

131 expansion is at least sufficient for drug screening, all as described below. 


\section{Early detection of tumor cells during culturing}

134 Tumors consist of a variety of different cell types. These include normal cell types that

135 can grow as well or even better in the provided culture conditions, possibly

136 outcompeting tumor cells (Dijkstra et al., 2020). It would therefore be useful to test for

137 the presence of tumor cells early during culturing to omit the unnecessary propagation

138 of cultures lacking any. At early time points, material is limited, impacting the range of

139 applicable assays. The establishment protocol therefore utilizes an RT-qPCR assay

140 after the first or second passage of cells with probes for standard RMS histopathology

141 markers, i.e., DES, MYOG, MYOD1 (WHO, 2020), and the fusion transcript in fusion-

142 positive RMS (FP-RMS) (Ponce-Castañeda et al., 2014). We considered a sample

143 positive for tumor cells if at least one of the three genes, plus for FP-RMS the fusion

144 transcript, test positive. All samples that successfully yield tumoroid models, show

145 positivity for at least one marker gene at this stage, while most models (17 out of 19)

146 are positive for all three marker genes and the fusion transcript if applicable (Fig. 2A

147 and B). The RT-qPCR-based approach is therefore a useful tool to determine

148 feasibility at an early stage.

RMS tumoroid models retain marker protein expression

151 A hallmark of RMS tumors is the expression of proteins associated with non-terminally

152 differentiated muscle (i.e., Desmin, Myogenin and MYOD1). Expression of these

153 proteins differs between RMS subtypes (Dias et al., 2000) and can be associated with

154 prognosis (Heerema-Mckenney et al., 2008). To properly reflect the original tumors,

155 RMS tumoroid models should therefore retain the expression patterns of these

156 proteins. The RMS tumoroid establishment protocol therefore includes a

157 morphological (H\&E) and immunohistochemical (IHC) assessment at the time of 
successful establishment (i.e., drug screening). To enable comparison between tumoroid models and the tumors they were derived from, models are grown as three-

160 dimensional spheres to mimic the three-dimensional architecture of tumors (Fig. 2C).

161 When evaluated in this manner, RMS tumoroid models show retained expression

162 levels and patterns of Desmin, Myogenin and MYOD1 at the time of successful establishment. Cellular morphology is also retained, with aRMS models displaying homogenous, primitive cells, with large nuclei and minimal cytoplasm, and eRMS models displaying more heterogenous tumor cells with variable maturation (Fig. 2C and Suppl. Fig. 1), as in the tumors (WHO, 2020). In summary, RMS tumoroid models

167 retain histopathological hallmarks of RMS tumors, giving a first indication that they reflect the tumors they were derived from to a high extent.

A collection of RMS tumoroid models that represent the diverse clinical presentation of RMS

172 Having established protocols for the acquisition, processing, initial growth, and 173 characterization of RMS tumoroid models, we applied this to 46 consecutive samples

174 from pediatric RMS patients treated in the Netherlands, resulting in a collection of 19 RMS tumoroid models ( $41 \%$ efficiency). These models are derived from tumors comprising both main histological subtypes (embryonal and alveolar), different fusion

177 types (fusion positive PAX3-FOXO1, PAX7-FOXO1, a novel fusion PAX3-WWTR1, as

178 well as fusion negative), various risk groups, locations, and are derived from primary 179 as well as relapsed disease (Fig. 1B and Suppl. Table 1). Outgrowth of cells from 180 highly aggressive RMS subtypes shows a higher success rate, as indicated by $83 \%$ 181 successful establishment for FP-RMS versus $16 \%$ for FN-RMS, and $61 \%$ success in 182 relapsed, versus $30 \%$ in primary RMS tumors, respectively. There was no statistically 
significant difference in the success rate between recently treated and untreated

184 tumors (33\% versus $43 \%$, respectively, $p=0.59$, chi-square test). Similar differences between relapsed and primary tumors in establishment rates have been observed for orthotopic PDX models (O-PDX) of RMS (Stewart et al., 2017). Compared to O-PDX

187 models of RMS, the overall success rate is lower for establishing RMS tumoroid models $(41 \%$ versus $65 \%$, respectively). While the success rate is lower, RMS tumoroid models can be more rapidly established. Engraftment of O-PDX RMS models takes 1 to 5.5 months before tumor growth is first observed in mice, while RMS tumoroid models can be fully established, i.e., characterized and subjected to drug screening, in as little as 27 days for highly aggressive tumors. Overall, the median time

193 from acquisition of the tumor sample to successful drug screening was 81 days (with

1947 models being screened in less than 2 months). This indicates that our approach could potentially be applied in a personalized medicine setting where it is crucial to obtain results as fast as possible to provide information on treatment options.

198 A subset of the tumoroid models were derived from the same patient, but at different points during treatment and/or from different body sites (marked with one or more asterisks in Fig. 1B). This potentially facilitates studies of tumor evolution or acquired treatment resistance. Furthermore, the collection contains a model of an eRMS with a novel fusion between PAX3 and WWTR1. Such fusions have been reported as rare events in biphenotypic sinonasal sarcomas, which usually harbor PAX3-MAML3 fusions (Loarer et al., 2019). Taken together, the protocol efficiently yields tumoroid models from highly aggressive as well as from extremely rare RMS subtypes, resulting in an initial collection covering a broad spectrum of subtypes. 
RMS tumoroid models molecularly resemble the tumor they are derived from

209 Given that the present tumoroid collection is the first to be established from tumors of

210 purely mesenchymal origin, we asked to what extent the models further resemble the

211 tumors they were derived from besides the retained hallmark protein expression levels

212 and patterns described above. To this end, whole-genome sequencing (WGS) and

213 bulk mRNA sequencing (RNA-seq) of the tumor and tumoroid model at the time of

214 drug screening (i.e., successful establishment), were performed.

216 Copy number profiles were first compared between tumors and tumoroid models

217 showing that profiles are indeed highly concordant (Fig. 3A). The detected copy number alterations included those commonly observed in RMS, with genomic gains in

219 chromosome 8 in FN-RMS and gains in chromosome 1 and 12 in FP-RMS (Shern et al., 2014; Weber-Hall et al., 1996). In addition, copy number profiles of individual RMS tumors and derived tumoroid models show a high concordance (Suppl. Fig. 2A and B). This indicates that the established models resemble the tumors they were derived from on a more global genomic level.

Various mutational processes are active in cells, which cause distinct somatic mutational signatures. These signatures are characterized by specific patterns of single base substitutions (SBS) in the context of their two flanking bases (Alexandrov et al., 2020). The presence of certain somatic mutational signatures in a cell can be associated with the underlying mutational processes. These processes are not restricted to in vivo systems, but can also occur during culture (Petljak et al., 2019),

231 forming a potential source of genomic destabilization. To test whether the somatic 232 mutational signatures and thus the underlying mutational processes present in the 
tumors ("T") are concordant with those in the derived tumoroid models ("O"), we first

234 measured the relative contributions of different signatures per sample. The main signatures observed are signatures associated with aging (SBS1 and SBS5), as well as a signature associated with increased oxidative stress (SBS18) (Alexandrov et al., 2020), and a signature associated with exposure to the chemotherapeutic Temozolomide (TMZ) (Kucab et al., 2019) used in the treatment of RMS (Defachelles et al., 2021) (Fig. 3B). Importantly, the signatures detected in the tumoroid models are highly concordant with those detected in the original tumors (Fig. 3B). In line with this, no global differences in SBS profiles between RMS tumors and derived tumoroid models are detected when analyzed collectively (Suppl. Fig. 2C). Lastly, we calculated the similarities of the individual SBS profiles of all tested tumor and tumoroid model samples. Tumoroid models cluster with the tumors they are derived from, indicating that the mutational landscape is retained in the models (Suppl. Fig. 2D). Only the tumor and tumoroid model of RMS127 do not cluster closely, likely due to derivation from a bone marrow aspirate with low tumor cell infiltration $(5-10 \%$ as estimated by pathology). Overall, there is a high concordance between the somatic mutational signatures of RMS tumors and the derived tumoroid models, again illustrating that the established models resemble the patient tumors.

While FP-RMS are driven by the prototypical fusion genes, FN-RMS are characterized by SNVs in known oncogenes such as TP53 or RAS family members (Shern et al., 2014). Currently available preclinical models of RMS do not cover the full spectrum of these SNVs, thus limiting the applicability to test novel targeted drugs in RMS. To investigate whether the established RMS tumoroid models harbor any of these SNVs and whether these were already present in the tumor, all SNVs predicted to be 
pathogenic for protein function and with a variant allele fraction (VAF) of above 0.3 in either tumor and/or tumoroid were evaluated. As already indicated by the mutational signature analyses, RMS tumoroid models retain SNVs present in the tumor to a high degree (Fig. 3C). Similarly, samples from the same patient but from different sites or acquired from different relapses also show a high overlap in SNVs (Fig. 3C).

264 Two of the FN-eRMS tumoroid models harbor previously described oncogenic mutations in FN-eRMS, i.e., CTNNB1 (p.T41A) in RMS012 and FGFR4 (p.V550L) in RMS444 (Shern et al., 2014). To our knowledge, these are the first preclinical RMS models harboring these specific mutations. Moreover, one PAX3-FOXO1 FP-aRMS (RMS410) displays an oncogenic mutation in KRAS (p.G12A), which is uncommon in FP-RMS (Shern et al., 2014). Lastly, two FN-RMS tumoroid models (RMS007 and RMS012) display non-annotated frameshift mutations in the BCOR gene with a high VAF of above 0.9. Mutations in BCOR have been reported in RMS with a higher prevalence in FN-RMS compared to FP-RMS (Shern et al., 2014). The mutation analysis shows that RMS tumoroid models not only retain specific SNVs already present in the tumor but that these models also contain mutations for which no preclinical model was previously available.

mRNA expression profiles of FN- and FP-RMS are fundamentally different, mainly due

278 to the transcriptome-wide impact of the fusion transcript in FP-RMS (Wachtel et al., 2004). Additionally, the transcriptional program of in vitro cultured organoids is influenced by the culture conditions and can deviate from the transcriptional program

281 of the parent tissue (Lu et al., 2021). Given that FN-RMS and FP-RMS tumoroid 282 models are cultured in the same medium, we asked whether the transcriptional 
differences observed between the original tumors are retained in the models. Analysis

previously been shown for primary RMS tumor samples (Wachtel et al., 2004).

287 Furthermore, correlation between the expression profiles of RMS tumor and tumoroid models of the same fusion-type is high, while correlation with control kidney tumors is low, showing that the fundamental differences in the expression profiles of FN- and FP-RMS are retained in culture (Fig. 3D). In summary, the early tumor cell detection by RT-qPCR, the morphological and marker protein analyses, WGS for copy number profiles, for somatic mutational signatures and for individual SNVs, as well as the comparative transcriptomic analyses, indicate that the RMS tumoroid models resemble the original RMS tumor they are derived from.

Genetic and transcriptional stability of tumoroid models over time

297 We next asked whether the models remain genetically and transcriptionally stable after

298 culture over extended periods. Although convenient for certain experimental approaches, conventional RMS cell lines can acquire new mutations through culturing. An example is the widely used PAX3-FOXO1 FP-aRMS cell line RH30, that harbors a TP53 mutation, uncommon in primary FP-RMS (Felix et al., 1992; Shern et al., 2014).

302 To investigate stability, a subset of RMS tumoroid models were kept in culture over

303 three to six months until they reached passage 40 ("OL" for late passage, as compared 304 to "O", the standard passage analyzed here and sufficient for drug screening) and characterized again by WGS and RNA-seq. Furthermore, two independently derived tumoroid models (i.e., established from the same tumor piece of which a part was cryo- 
preserved after the first tumoroid establishment) were included in this comparison ("O2") to assess the robustness of the establishment protocol.

310 RMS tumoroid models show long-term propagation potential with five out of seven

311 lines tested reaching passage 40 and two lines dropping out at passage 17 and 20,

312 respectively. As drug screening is usually performed between passage 6 to 12 , this

313 shows that models can be readily used after such an initial screening. Comparison of

314 individual copy number profiles of standard and late passage (Suppl. Fig. 3A), as well

315 as independently derived paired tumoroid models (Suppl. Fig. 3B) shows no major

316 copy number differences between the respective models. Furthermore, analysis of

317 somatic mutational signatures shows that the contributions of identified somatic

318 mutational signatures (Fig. 4A) as well as the contributions of individual SBSs between

319 models (Suppl. Fig. 3C) are highly similar. Lastly, analysis based on SBS profiles

320 shows clustering of models derived from individual patients (Suppl. Fig. 3D). The

321 exception to this is RMS335 "OL" which may be due to a mutation in the DNA damage

322 response gene $A T R$ in both the tumor and the tumoroid sample, potentially resulting

323 in the gain of new mutations during culturing. The similarity between SBS profiles of

324 RMS335 "O" and "OL" was nevertheless high. Taken together, these analyses show

that RMS tumoroid models generally remain stable, even over extended periods of

326 culturing and that the establishment protocol is robust, yielding highly similar models

327 when independently derived from a single tumor sample.

329 As before (see Fig. 3C), individual pathogenic SNVs were also evaluated. Importantly,

330 the majority of SNVs are retained after long-term culturing, with the models acquiring

331 only a few additional SNVs (Fig. 4B). In addition, the models independently derived 
332 from the same tumor show a high overlap of pathogenic SNVs (Fig. 4B). Notably, the

333 afore-mentioned oncogenic mutations in CTNNB1 and FGFR4 are detected in all

334 related samples (Fig. 4B). This further indicates that the models do not lose key

335 mutations and that the establishment protocol results in the outgrowth of

336 representative tumor cells harboring these mutations. RNA-seq expression profiles

337 from the standard passage, late passage, as well as the independently derived

338 tumoroid models were compared to test whether the core transcriptional program of

339 RMS is retained. Principal component analysis shows that global expression profiles

340 of tumoroid models derived from the same patient cluster together (Fig. 4C). This

341 suggests that our models are not only genetically, but also transcriptionally stable over

342 time.

RMS tumoroid drug screening reflects established drug sensitivities

345 Having established that the RMS tumoroid models resemble the tumors they are

346 derived from with stability during prolonged culturing, their suitability for research was

347 further investigated in two specific ways, i.e., via drug screening and CRISPR/Cas9

348 genome editing. Development of novel RMS treatments has been hampered by a lack

349 of preclinical models that can efficiently be subjected to drug screening. To be of use

350 for such screening approaches, it is imperative that new models reflect drug

351 sensitivities already known for these tumors. This was investigated with a custom

352 pediatric cancer library of 165 compounds, comprising standard of care

353 chemotherapeutics as well as early-phase clinical trial targeted compounds. To mimic

354 the three-dimensional configuration of tumors, cell plating was optimized so that

355 tumoroid cells form homogenous 3D spheres in 384-well plates. The protocol also

356 included prior growth curve determination of the number of cells that must be plated 
for each individual tumoroid model to prevent overgrowth during screening (Materials

\& Methods).

360 Vincristine and actinomycin D are routinely used in RMS treatment and indeed show

361 broad efficacy in all models tested (Fig. 5A, bottom highlighted box). Furthermore, the

362 proteasome inhibitor bortezomib shows a similarly high efficacy in all tested models.

363 This is in line with previous studies showing that both major histological RMS subtypes

364 are susceptible to bortezomib treatment in vitro (Bersani et al., 2008) and in vivo

365 (Manzella et al., 2020), suggesting that RMS tumoroid models indeed reflect drug

366 sensitivities known for RMS tumors.

368 Clustering of RMS tumoroid models based on drug efficacy shows two main groups, comprising 12 and 4 models, respectively, with one unclustered model (RMS000FLV).

This model nevertheless shows high sensitivity to the afore-mentioned drugs (bottom of Fig. 5A). The outlier behavior is not caused by differences in growth during the experiment (see Suppl. Table 2) and may be explained by the fact that this is the only treatment-naïve FP-aRMS model in the collection (Fig. 1B). The group of 4 models, that contains all successfully screened FN-RMS tumoroid models, is more sensitive to all tested inhibitors of MEK/ERK (MAPK signaling pathway) as well as the two

376 inhibitors of $\mathrm{Y}$-secretase ( $\mathrm{NOTCH}$ signaling pathway) in the drug panel, when 377 compared to the other group containing only FP-RMS tumoroid models (Fig. 5A, top 378 highlighted box, and Fig. 5B and C). Importantly, sensitivity of FN-RMS against 379 inhibitors of MAPK and NOTCH signaling has previously been reported (Belyea et al., 380 2011; Yohe et al., 2018). This group also contains the FP-aRMS tumoroid model 381 RMS000HQC which shows a very low expression of its fusion transcript (Fig. 2B), 
which potentially resulted in it displaying sensitivities more commonly observed in $\mathrm{FN}$ -

RMS. Taken together, our results indicate that drug sensitivities observed in RMS

these models hold for testing novel drugs. As drug screening could be performed as

early as 27 days after sample acquisition, with a median time to drug screening of 81

days, this highlights their relevance for personalized approaches.

RMS tumoroid models can be molecularly edited using CRISPR/Cas9

The applicability of preclinical models would be further enhanced by the possibility of genetic modification. To test this, we used CRISPR/Cas9 (Jinek et al., 2012) to knock out the well-known tumor suppressor gene TP53. This choice is based on the recent report that loss of functional P53 protein confers a worse prognosis in RMS (Shern et

WGS, were transfected with a plasmid harboring a TP53 targeting sgRNA as well as

Cas9. Successfully edited cells were selected with nutlin-3 (Drost et al., 2015) (Fig.

$3976 \mathrm{~A}$ ), resulting in cells with complete loss of P53 protein as confirmed by Western Blot

398 (Fig. 6B). Sanger sequencing shows a spectrum of Indels consistent with a polyclonal population of P53 deficient cells (Suppl. Fig. 4A).

P53 deficient eRMS tumoroid cells are more sensitive to the checkpoint kinase inhibitor prexasertib

In light of the recently reported prognostic significance of P53 loss in RMS (Shern et various cellular functions, including response to DNA damage through control of the 
407 give cells time to repair DNA damage (Yonish-Rouach et al., 1991). The rate of DNA 408 double-strand breaks (DSBs) was therefore first examined in the P53 deficient RMS

409 tumoroid cells by measuring phosphorylation of histone H2AX serine 139, a proxy for

410 DNA DSBs (Rogakou et al., 1998). No difference in the amount of DNA DSBs was

411 observed between TP53 wildtype and knockout cells however (Suppl. Fig. 4B). To

412 avoid catastrophic failure of the proliferative machinery and avoid cell death, P53

413 deficient cells are dependent on the G2/M checkpoint to repair DNA DSBs

414 (Dobbelstein and Sørensen, 2015). Inhibitors of the checkpoint kinase 1 (Chk1), which

415 controls the G2/M checkpoint, have therefore been suggested as a treatment for P53

416 deficient tumors, such as in certain ovarian and breast cancer subtypes (Lee et al.,

417 2018; Ma et al., 2012). This hypothesis was tested, resulting in the observation that

418 TP53 knockout RMS tumoroid cells are significantly $(p=0.008)$ more sensitive to the

419 Chk1 inhibitor prexasertib, compared to their wildtype counterpart (Fig. 6C). The 420 absolute difference between $\mathrm{IC}_{50}$ values is not extreme (18.5 nM for TP53 wildtype 421 versus $12.9 \mathrm{nM}$ for TP53 knockout cells), likely reflecting the fact that RMS012 TP53 422 wildtype cells are already sensitive to prexasertib. These results indicate that 423 exploiting replicative stress in P53 deficient RMS is indeed an interesting therapeutic 424 avenue to pursue. Moreover, the results demonstrate that it is possible to perform 425 gene editing in these novel RMS tumoroid models, thereby further increasing their 426 utility. 


\section{Discussion}

A tumoroid collection of purely mesenchymal origin

430 To date, organoid technology has primarily been employed to generate models of

431 malignant tumors of epithelial origin (i.e., carcinomas) (Bleijs et al., 2019). Feasibility

432 to use this technology on non-epithelial cancer has only recently been shown

433 (Abdullah et al., 2021; Fusco et al., 2019; Jacob et al., 2020; Saltsman et al., 2020;

434 Yamazaki et al., 2021). Here we extend the tumor organoid approach, demonstrating applicability to tumors of entirely mesenchymal origin (i.e., sarcomas), resulting in only the second thoroughly characterized tumoroid collection specific for pediatric cancer.

438 Two factors likely contributed to the delayed adaptation of organoid technology to sarcomas. First, the technology was developed for healthy epithelial tissue, followed later by translation to the corresponding cancer entity, as in the case of colorectal carcinoma (Sato et al., 2009, 2011; Van De Wetering et al., 2015). The cell of origin

442 of RMS is still not fully characterized (Hettmer and Wagers, 2010). Therefore, extensive culture optimization of healthy tissue first, with translation to cancerous tissue later, has not been feasible for RMS. Consequently, optimization had to be to the issue of tissue availability, sarcomas are far less common than carcinomas,

447 accounting for less than $1 \%$ of all solid adult malignancies (Burningham et al., 2012).

448 In pediatric cancer however, sarcomas are much more common, encompassing $21 \%$ 449 of all solid tumors in children (Burningham et al., 2012). This further highlights the 450 importance of the currently described approach and collection. 
453 The tumoroid models include representatives of both major histological subtypes, all

454 major fusion types, different age groups, both sexes, treatment-naïve as well as pre455 treated, primary as well as metastatic tumors (Fig. 1B and Suppl. Table 1). Rarer 456 subtypes such as sclerosing/spindle-cell RMS (Rudzinski et al., 2015) will be exciting 457 to include, as also indicated by a recent case report (Acanda De La Rocha et al., 458 2021). Compared to patient incidence rates (Glosli et al., 2021), the collection has an 459 underrepresentation of head and neck RMS. Although RMS tumor samples from this 460 region were acquired, models from such samples failed, regardless of subtype, clinical 461 stage, sample quantity or quality. Interestingly, this indicates that RMS arising in the 462 head and neck may depend on specific factors that have not yet been identified. 463 Studies in genetically engineered mice indicate that aberrant Hedgehog signaling can 464 give rise to FN-RMS from non-myogenic endothelial progenitors in the head and neck 465 (Drummond et al., 2018). Here, activation of Hedgehog signaling by Smoothened 466 agonists did not facilitate establishment of head and neck RMS tumoroid models, 467 indicating that additional factors may be necessary. Regardless of such future 468 developments, the protocol described here yields models that can be rapidly established from a broad range of quite different RMS subtypes.

New models for RMS research

472 Currently available preclinical models of RMS include conventional cell lines, various 473 genetically engineered animal models (GEMs), as well as patient-derived xenograft models (PDX or O-PDX when transplanted orthotopically) (Imle et al., 2021; Kashi et

475 al., 2015). Each of these systems possesses specific benefits and drawbacks, 476 resulting in suitabilities for different research questions or stages in drug development 477 (Kim et al., 2020). While conventional cell lines are easy to use, with low costs, large- 
scale screening potential and ease of genetic modification, due to prolonged culturing they often do not recapitulate many basic features of the genetic and molecular background of the tumor they were derived from, thus possessing only limited predictive value (Hinson et al., 2013). GEMs, on the other hand, are well-suited for cell of origin studies and can provide valuable insights into cancer onset mechanisms. GEMs are usually not suitable for high-throughput screening, mostly due to the low tumor penetrance or the intricacies of animal studies (Kersten et al., 2017). In contrast to GEMs, in O-PDX models, tumor samples are transplanted onto immune-deficient mice to allow for engraftment, growth and later propagation of the tumor tissue from mouse to mouse. Tumors propagated in this manner are thought to be genetically stable over time and to reflect the patient tumor they were derived from, giving them predictive value concerning preclinical drug testing (Gao et al., 2015). Disadvantages include the necessary use of mice as hosts and the tumor-entity specific engraftment time which can take up to several months (Pompili et al., 2016). Furthermore, there is evidence that not all PDX models are genetically stable (Petljak et al., 2019).

The RMS tumoroid models combine several of the above-mentioned benefits. They can be rapidly established and expanded like conventional cell lines, enabling drug screening. As with cell lines, molecular editing to mimic certain disease backgrounds

497 is possible using CRISPR/Cas9. Unlike cell lines however, tumoroid models depend 498 on defined media including recombinant growth factors as well as an ECM substitute, resulting in higher costs. RMS tumoroid models molecularly resemble the patient tumor they are derived from, sharing this characteristic with O-PDX models, while 501 possessing the above-mentioned advantages of rapid establishment and expansion.

502 Compared to O-PDX models, establishment success is lower in RMS tumoroid 
models, indicating that niche factors in the host mice are important for facilitating establishment. This may be especially important in particular subtypes such as RMS

505 from the head and neck region. On the other hand, RMS tumoroid models are less

506 intricate in their propagation. Lastly, while GEMs are considered essential for cell of

507 origin studies, recent advances have shown that by genetic editing, such studies can

508 now also be performed in tumoroid models (Custers et al., 2021).

510 In conclusion, we established a well-characterized, well-annotated collection of RMS

511 tumoroid models, being the first such collection of tumoroid models derived from purely

512 mesenchymal malignant tumors (i.e., sarcomas) and only the second comprehensive

513 tumoroid model collection derived from pediatric cancer (Calandrini et al., 2020). This

514 collection contains all major subtypes of RMS and the models can be used for drug

515 screening as well as molecular editing. An interactive, browser-based companion

516 Shiny app (https://rmsdatabiobank.shinyapps.io/app1_3/) that makes all the described

517 data easily accessible, accompanies this paper. The RMS tumoroid models will be a

518 useful complementary system to study the biology of RMS and to improve treatment. 
520 Acknowledgements: We thank the patients and their parents for contributing by consenting to this study; the nurses, doctors and other health care professionals in our

522 institute with whom we work closely; our center's high-throughput screening facility, in particular Bianca Koopmans, Kimberley Ober, and Sander van Hooff; Philip Lijnzaad,

524 Thanasis Margaritis and Tito Candelli, as well as past and present members of the 525 Holstege group.

Financial support: M.M. received financial support from the Deutsche

Forschungsgemeinschaft (\#408083583). C.C. was supported by Foundation Children

529 Cancer Free (\#292). J.D. received support from the European Research Council 530 (ERC) starting grant 850571 and the Dutch Cancer Society (KWF)/Alpe d'HuZes Bas Mulder award (\#10218). We are grateful for the financial support provided by the Foundation Children Cancer Free (KiKa core funding).

Author contributions: Conceptualization, M.M. and F.H.; Methodology, M.M., M.G.K. and W.B.; Investigation: M.M., M.G.K, T.d.S., W.B., E.F-M., M.B., J.D., C.C. and F.M., M.v.T.; Resources, M.D., S.E., K.L., R.K., S.T.v.S., L.H., U.F., J.M., M.v.N., B.T., J.HK., P.K., J.M., M.v.d.W., R.v.B. and J.D..; Data curation, M.G.K., T.d.S., A.J. and H.K.; Writing - Original Draft, M.M. and F.H.; Visualization, M.M., T.d.S. and F.M.; Funding 539 Acquisition, F.H., Supervision, M.M. and F.H.

541 Conflict of interest disclosure statement: The authors declare no competing 542 interests. 


\section{Figure legends}

546 Fig. 1 - A collection of RMS tumoroid models that represent the diverse clinical

547 presentation of RMS: (A) Tumor organoid (tumoroid) pipeline. WGS = whole-genome

548 sequencing, RNA-seq = mRNA sequencing, liq N2 = liquid nitrogen. (B) Overview of

549 available RMS tumoroid models in the collection separated by primary vs. metastatic

550 site and exact tumor location. The color of the inner circle indicates the histological

551 subtype while the color of the outer circle indicates the presence or absence of a fusion

552 transcript. Letters within the circle indicate disease instance. Asterisks mark tumoroid

553 models derived from the same patient but from distinct tumor samples. (C) Brightfield

554 microscopy images of two representative RMS tumoroid models from a fusionnegative embryonal and a PAX3-FOXO1 fusion-positive alveolar tumoroid model grown in a two-dimensional monolayer in two magnifications as indicated by the scale bars.

Fig. 2 - Early detection of tumor cells during culturing and retained marker

protein expression: (A) RT-qPCR of early passage RMS tumoroid models shows positivity for at least one gene used in standard-of-care pathology analysis (DES, MYOG, or MYOD1). Conventional RMS cell lines (RD and RH30) were used as positive controls, while two Synovial Sarcoma (SS000DAZ and SS077) tumoroid

564 models were used as negative controls. Gene expression was normalized to the expression of a house-keeping gene and human reference RNA (HREF) via the $\triangle \Delta \mathrm{Cq}$ method. (B) RT-qPCR of early passage RMS tumoroid models reliably detects the

567 aberrant fusion transcripts. Fusion gene expression was normalized to the expression 568 of a house-keeping gene via the $\Delta \mathrm{Cq}$ method. (C) Morphological (via $\mathrm{H} \& \mathrm{E}$ ) and 
immunohistochemical (IHC) comparison of RMS tumors and derived RMS tumoroid models shows retained marker protein (Desmin, Myogenin and MYOD1) expression and cellular morphology. Scale bars equal $200 \mu \mathrm{m}$.

Fig. 3 - RMS tumoroid models molecularly resemble the tumor they are derived

from: (A) Copy number frequency plots of RMS tumors (upper row) and derived RMS tumoroid models (lower row) divided by fusion-status (columns). Chromosomes are annotated on the $x$-axis from left to right while the y-axis shows the percentage of samples in this group carrying a gain (red) or loss (blue) in this genomic region. (B)

578 Contribution of somatic mutational signatures per tumor and tumoroid model. $\mathrm{SBS}=$

579 single base substitution, $\mathrm{TMZ}=$ temozolomide, $\mathrm{T}=$ tumor, $\mathrm{O}=$ tumoroid (C) Table depicting pathogenic single-nucleotide variants (SNVs) in RMS tumors (T) and tumoroid models $(\mathrm{O})$. Circle color indicates SNV type while circle size indicates variant allele fraction (VAF). Vertical dotted lines separate samples derived from individual patients. Highlighted are genes previously reported for this RMS subtype. (D)

584 Correlogram of bulk mRNA sequencing expression profiles of pediatric kidney tumors (controls) as well as RMS tumoroid models and RMS tumors. CCRCC $=$ Clear Cell Renal Cell Carcinoma, $\mathrm{CMN}=$ Congenital Mesoblastic Nephroma, $\mathrm{WT}=$ Wilms Tumor, Cor $=$ correlation .

Fig. 4 - Genetic and transcriptional stability of tumoroid models over time: $(A)$

590 Contribution of somatic mutational signatures per tumor and tumoroid model. SBS = single base substitution, $\mathrm{TMZ}=$ temozolomide, $\mathrm{T}=$ tumor, $\mathrm{O}=$ standard passage

592 tumoroid model, $\mathrm{OL}=$ late passage tumoroid model, $\mathrm{O} 2$ = independently derived

593 tumoroid model. (B) Table depicting pathogenic single-nucleotide variants (SNVs) in 
different RMS tumoroid models derived from the same tumor sample. Circle color tumoroid model while the symbol indicates the sample. $\mathrm{O}=$ standard passage tumoroid model, $\mathrm{OL}=$ late passage tumoroid model, $\mathrm{O} 2$ = independently derived tumoroid model.

603 Clustered heatmap of viability measurements per RMS tumoroid model ( $x$ axis) and drug (y axis), showing the Area Under the Curve (AUC) after treatment of the cells for $120 \mathrm{~h}$ with a dose-range of $0.1 \mathrm{nM}$ to $10 \mu \mathrm{M}$. Low AUC (red) indicates high drug efficacy while high AUC (blue) indicates low drug efficacy. Annotated clusters of (1) MEK/ERK and $\mathrm{Y}$-secretase inhibitors showing specific efficacy in RMS tumoroid models without (RMS007, RMS012, RMS444) or low (RMS000HQC) fusion transcript expression, and (2) drugs that show broad efficacy across RMS tumoroid models. (B)

610 Principal component analysis of drug screening AUC values of the RMS tumoroid models (RMS000FLV omitted due to outlier behavior as discussed in the main text).

612 The ellipse indicates the cluster that shows specific sensitivity against MEK/ERK and

613 Y-secretase inhibitors. (C) Principal component analysis of the top 25 contributing 614 drugs that influence variance. The ellipse indicates the MEK/ERK and $\mathrm{Y}$-secretase 615 inhibitors as well as AZD4547 (RMS000FLV omitted as described above). 
619 prexasertib: (A) Transfection and selection strategy to achieve TP53 knockout in a

620 TP53 wildtype tumoroid model. (B) Western Blot analysis of TP53 wildtype (WT) and

621 knockout (KO) RMS tumoroid line RMS012. Histone 3 (H3) served as loading control.

622 (C) Dose-response curve of TP53 WT and KO cells treated with the Chk-1 inhibitor

623 prexasertib. Thin lines with numbers indicate individual technical replicates while thick

624 lines indicate fitted lines over all replicates. 
Materials \& Methods

626

Tumor sample acquisition

628 Tumor samples of RMS were obtained via an established tumor sample acquisition

629 route from patients treated at the Emma Children's Hospital Amsterdam (Amsterdam

630 UMC) (RMS006, RMS007, RMS013) or as part of the biobank initiative of the Princess

631 Máxima Center for Pediatric Oncology, Utrecht, Netherlands (PMC) (remaining tumor

632 samples). Ethics approval was granted for the biobanking initiative, and the PMC

633 biobank committee granted approval for the present project. All patients and/or their

634 legal representatives signed informed consent to have tumor samples taken for 635 biobank usage.

636 A subset of patients was furthermore enrolled in a local personalized medicine trial

637 (i.e., iTHER study) through which a subset of DNA and RNA specimens from RMS 638 tumors were obtained.

639

640 Tumor sample preparation for establishment of RMS tumoroid models

641 Solid tumor samples (i.e., needle biopsies or resection specimens) were transferred

642 to collection medium (see below) to retain viability of cells. After pathological 643 examination, suitable samples (i.e., containing tumor cells) were processed in a sterile 644 work environment as follows: the sample was transferred to a sterile dish and covered 645 with a droplet of $B M 1^{*}$ culture medium (see below) before being minced to fine pieces 646 using scalpels. If the tumor sample was of sufficient size, a portion of this minced 647 mixture was stored viably (see below). The remainder of the mixture was put on a pre648 wet $70 \mu \mathrm{M}$ strainer, scrapped with a cell scraper to obtain a single-cell suspension and 
649 collected in a tube (A, single-cell fraction, cultured in 2D). Tissue fragments left on the

650 strainer were collected in another tube (B, strainer fraction, cultured in 3D).

651 A: The single-cell fraction was now spun down $\left(300 \mathrm{~g}, 5 \mathrm{~min}, 4^{\circ} \mathrm{C}\right)$ and the supernatant

652 was removed from the resulting pellet. If the pellet was of grey color (i.e., not containing

653 a high percentage of red blood cells, RBCs), the pellet was resuspended in $1 \mathrm{ml}$ of

$654 \mathrm{BM} 1^{*}$ medium. If the pellet contained a high percentage of RBCs (i.e., by displaying a red stain), the cell pellet was resuspended in red blood cell lysis buffer (Roche) and incubated at room temperature for $5 \mathrm{~min}$. Thereafter, the reaction was stopped by adding collection medium and the mixture was spun down again (as above). Again, supernatant was removed and now the pellet was resuspended in $1 \mathrm{ml}$ of $\mathrm{BM} 1$ * medium (without Basement Membrane Extract, BME, see below). In each case, cells were now counted using a TC20 Automated Cell Counter (BioRad) to get a rough estimate on viability and cell numbers. Cells were plated with a sufficient density (at least 10.000 viable cells per $1 \mathrm{~cm}^{2}$ of surface area), supplemented with 0.3 to $0.5 \%$ cold BME type 2 (see below).

B: The strainer fraction was dissolved in pure cold BME (roughly one-third volume strainer fraction and two-third volume BME) and mixed thoroughly. Droplets of 5 to 10 $\mu \mathrm{l}$ of this mixture were formed on 24 - or 48-well pre-warmed cell culture plates and

667 incubated for $5 \mathrm{~min}$ at room temperature to allow the BME to solidify. Thereafter, the 668 cell culture plates were incubated upside-down for another $25 \mathrm{~min}$ at $37^{\circ} \mathrm{C}$ to allow 669 the material to "sink" to the top of the droplet. Upon completed solidification, BM1* 670 medium (at room temperature (RT) and without BME) was added to the wells so that 671 droplets were very fully submerged in medium.

672 In the case of bone marrow aspirates as tumor samples (RMS410 and RMS127), 673 sample tubes were spun down (300 g, $5 \mathrm{~min}, 4^{\circ} \mathrm{C}$, slow break) to separate RBCs and 
nucleated cells (white clot at the bottom of the tube). In the case of RMS410, the normal hematopoietic system was almost entirely superseded by infiltrating tumor cells so that the clot consisted mainly of tumor cells which could directly be plated (in BM1* with BME) which resulted in rapid outgrowth of the model. In the case of RMS127, the

678 percentage of infiltrating tumor cells was estimated by pathology to be low at circa 5 to $10 \%$. Therefore, initial cultures (plated in $\mathrm{BM} 1^{*}$ and $\mathrm{BME}$ ) from the white clot also contained normal nucleated bone marrow cells which, however, were eventually outcompeted by the rapidly growing tumor cells which overtook the culture.

\section{Tumoroid model culturing and propagation}

684 Growing RMS tumoroid models were inspected regularly and showed adequate growth behavior under conventional cell culture conditions (i.e., $37^{\circ} \mathrm{C}, 5 \% \mathrm{CO}_{2}$ ). All models were regularly tested negative for mycoplasma contamination. Estimated division times ranged from 24 to 72 hours for most models. Models were passaged once or twice per week at a confluency of 70 to $80 \%$. For passaging, old culture medium was aspirated and cells were briefly washed with sterile DPBS (Gibco, cat no. 14190144). Cell detachment was performed using TrypLE Express Enzyme (1X, phenol red, Gibco, cat no. 12605010). Depending on the model and the BME percentage, detachment took between 3 and 10 minutes (higher BME concentrations resulting in longer detachment time). Cells were collected by flushing the well or flask with collection medium and the resulting mixture was spun down $\left(300 \mathrm{~g}, 5 \mathrm{~min}, 4^{\circ} \mathrm{C}\right)$.

695 Thereafter, the supernatant was removed, and the pellet was resuspended in $1 \mathrm{ml}$ of 696 BM1* and cells were counted using a TC20 Automated Cell Counter (BioRad). Single697 cell suspensions were mostly re-seeded at the same density as their parental/previous 698 generation/passage. 
$700 \quad$ Cell culture media

701 Base medium (BM)

702 To prepare a $500 \mathrm{ml}$ bottle of BM, Glutamax (5 ml, Gibco, cat no. 35050061),

703 Penicillin/Streptomycin (10,000 U/ml, $5 \mathrm{ml}$, Gibco, cat no. 15140122), and B27 (without

704 vitamin A, $10 \mathrm{ml}$, Gibco, cat no. 12587010) were added to a full bottle of advanced

705 DMEM/F12 (500 ml, Gibco, cat no. 12634010). BM was stored at $4{ }^{\circ} \mathrm{C}$ and was used

706 within two months.

707

708 Complete culture medium $\left(B M 1^{*}\right)$

709 To prepare the complete culture medium BM1*, $47.5 \mathrm{ml}$ of the above-mentioned base medium (BM) was taken and pipetted into $50 \mathrm{ml}$ tube. Thereafter, the components

711 below were added (no specific order). BM1* was stored at $4{ }^{\circ} \mathrm{C}$ and was used within

7127 to 10 days (thereafter, the stability of the growth factors may be compromised).

714 Components:

$715 \quad \mathrm{~N} 2$

$716 \quad \mathrm{~N}$-acetylcysteine $(500 \mathrm{mM})$

717

718 Sodium pyruvate (100 mM)

719 Heparin $(5,000 \mathrm{U} / \mathrm{ml})$

720

721

722
MEM non-essential amino acids $500 \mu \mathrm{l}$

hEGF $(2 \mu \mathrm{g} / \mathrm{ml})$

hFGF-basic $(40 \mu \mathrm{g} / \mathrm{ml})$

hIGF1 $(100 \mu \mathrm{g} / \mathrm{ml})$
$500 \mu \mathrm{l}$

$5 \mu \mathrm{l}$

$500 \mu \mathrm{l}$

$125 \mu \mathrm{l}$

$500 \mu \mathrm{l}$

$50 \mu \mathrm{l}$

$10 \mu \mathrm{l}$
Gibco, cat no. 17502048

Sigma, cat no. A9165

Gibco, cat no. 11140035

Gibco, cat no. 11360070

Sigma, cat no. H3149-10KU

Peprotech, cat no. AF-100-15

Peprotech, cat no. 100-18B

Peprotech, cat no. 100-11 
723 RKi (Y-27632, $100 \mathrm{mM})$

724

A83-01 (5 mM)
$5 \mu l$

$50 \mu \mathrm{l}$
AbMole Bioscience, cat no. M1817

Tocris Bioscience, cat no. 2939

Collection medium

727 To prepare a $500 \mathrm{ml}$ bottle of collection medium, Glutamax $(5 \mathrm{ml}$, Gibco, cat no. 35050061), Penicillin/Streptomycin (10,000 U/ml, 5 ml, Gibco, cat no. 15140122), and HEPES (1 M, $5 \mathrm{ml}$, Gibco, cat no. 15630049) were added to a full bottle of advanced and was used within two months.

\section{Basement Membrane Extract type 2 (BME)}

734 To facilitate attachment of cells, culture medium was supplemented with 0.1 to $0.5 \%$ product, resulting occasionally in suboptimal attachment of cells when the BME concentration was too low. On average, 0.2 to $0.3 \%$ BME supplementation was sufficient for stably established RMS tumoroid models. However, upon encountering suboptimal cell attachment, BME concentrations were raised to $0.5 \%$ in established cultures. During the initial establishment process, higher concentrations of BME $(0.3$ $\%$ to $0.5 \%$ ) showed increased attachment rates of cells.

\section{Freezing and storing procedure}

745 RMS tumor samples as well as established RMS tumoroid cultures were viably frozen

746 as follows: a sufficient number of viable cells (at least $0.5 \times 10 \mathrm{E} 6$, mostly $1 \times 10 \mathrm{E} 6$ ) were

747 diluted in $0.5 \mathrm{ml} \mathrm{BM1*}$ medium in a cryo tube. Then, an equal volume of freeze-mixture 
consisting of $80 \%$ fetal calf serum (FCS) and $20 \%$ DMSO was added dropwise, resulting in final concentrations of $50 \% \mathrm{BM}^{*}$ medium, $40 \% \mathrm{FCS}$, and $10 \% \mathrm{DMSO}$. long term storage, frozen vials were transferred to liquid nitrogen.

\section{Thawing procedure}

754 Cryo-preserved RMS tumoroid cell suspensions were quickly defrosted in a water bath volume to dilute the DMSO). Samples were then spun down $\left(300 \mathrm{~g}, 5 \mathrm{~min}, 4{ }^{\circ} \mathrm{C}\right)$, supernatant was removed, and the cell pellet was resuspended in $1 \mathrm{ml}$ of $\mathrm{BM} 1$ * medium. Thereafter, cells were counted using a TC20 Automated Cell Counter (BioRad). Cell viability was on average 20 to $50 \%$ lower compared to viability at the time of freezing. Cells were then plated at a proper density in BME-supplemented $\mathrm{BM} 1^{*}$ medium and needed one to two weeks to recover before being stable enough for further experiments. Restarting tumoroid cultures from cryo vials was possible for all tumoroid models.

\section{Early tumor validation by RT-qPCR}

766 Early during tumoroid establishment (upon first or second passaging), a portion of cells

767 was set aside for evaluation of marker gene expression. For this, cells were spun down

768 (500 g, $\left.5 \mathrm{~min}, 4^{\circ} \mathrm{C}\right)$, the supernatant was removed from the pellet, the pellet was dissolved in Trizol (10 minutes incubation at RT) and was immediately processed or snap-frozen and stored at minus $80{ }^{\circ} \mathrm{C}$ until further processing. Upon processing, first

771 the organic and aqueous phases were separated by addition of $20 \%$ chloroform,

772 followed by spinning down (maximum centrifugation speed, $15 \mathrm{~min}, 4^{\circ} \mathrm{C}$ ). The (upper) 
aqueous phase was further processed using the Direct-zol RNA MiniPrep Kit (Zymo Research) according to the manufacturer's protocol, including the recommended DNAse I treatment. Quality and quantity of isolated RNA were measured using a NanoDrop OneC (Thermo Fisher Scientific). Synthesis of cDNA from isolated RNA as well as a Universal Human Reference RNA that was used as negative control or for normalization (HREF, Stratagene/Agilent \# 740000) was performed using an oligo-dT primed SuperScript III Reverse Transcriptase (Invitrogen) based reaction according to the manufacturer's protocol. RT-qPCR was performed with the obtained cDNAs testing for expression levels of G6PD (forward: 5'-ACGGCAACAGATACAAGAAC-3', reverse: 5'-CGAAGTGCATCTGGCTCC-3'; product size: 86 bp) (Amary et al., 2007), DES (forward: 5'-CCGTGGTCTCTTACTTTCCTTT-3', reverse: 5'CCCACTTTCTCTCСTTCTCAATC-3'; product size: 119 bp), MYOG (forward: 5'TGCCCAACTGAGATTGTCTTC-3', reverse: 5'-CTGCTACAGAAGTAGTGGCATC3', product size: 81 bp), MYOD1 (forward: 5'-GTAGCAGGTGTAACCGTAACC-3', reverse: 5'-CACACCATGCCTCAGAGATAAA-3', product size: $148 \mathrm{bp}$ ), the PAXFOXO1 fusion transcript (forward: 5'-CCGACAGCAGCTCTGCCTAC-3', reverse: 5'TGAACTTGCTGTGTAGGGACAG-3', product size: 171 bp for PAX3-FOXO1 and 159 bp for PAX7-FOXO1) (Ponce-Castañeda et al., 2014) as well as the PAX3-WWTR1 fusion transcript (forward: 5'-AGCACCAGGCATGGATTT-3', reverse: 5'TTCGAGGTCTGTGTCTAGGT-5', product size: $192 \mathrm{bp}$ ). Expression levels of DES, MYOG, MYOD1, and PAX-FOXO1 were normalized to G6PD (housekeeping gene) and referenced to the corresponding expression levels in the HREF using the $\triangle \triangle \mathrm{Cq}$ method (for the fusion transcript only normalization to G6PD as the lack of a fusion expression in HREF did not permit a further reference step). 
To perform IHC, tumoroid models were grown as floating 3D spheres. For this, 1days, depending on the growth characteristics of the respective tumoroid model. supernatant was removed, and the sphere-containing pellet was resuspended in cold PBS to wash off any remaining medium. The mixture was again sedimented (see above), and PBS was aspirated. Spheres were now fixed using formalin $10 \%(\mathrm{v} / \mathrm{v}),(=$ $4 \%$ (w/v) HISTO GRADE, neutralized (pH $7.0 \pm$ 0.2), J.T. Baker, 3933.9020 VWR) for were then washed twice with PBS and dehydration was performed by adding ethanol

812 solutions with increasing percentages (25 \% EtOH for $15 \mathrm{~min}, 50 \% \mathrm{EtOH}$ for $15 \mathrm{~min}$,

$81370 \% \mathrm{EtOH}$ for $15 \mathrm{~min}$ - after this step, spheres were stored at $4{ }^{\circ} \mathrm{C}$ and further 814 processed in batches). Now, spheres were stained with $0.8 \mathrm{~g} / \mathrm{l}$ Eosin $\mathrm{Y}$ dissolved in 96 \% EtOH (Sigma, E4009) for 30 min and subsequently incubated three times with 100 $\%$ EtOH for 30 min each. Thereafter, spheres were incubated in n-Butanol (three times

$81730 \mathrm{~min}$ ) and melted paraffin (three times) before they were Paraffin-embedded using 818 the HistoCore Arcadia H (Leica Biosystems) following the manufacturer's protocol in

819 a medium size mold. Hardened paraffin blocks were cut into $4 \mu \mathrm{m}$ slices using a 820 microtome (HM 355S Automatic Microtome, Thermo Scientific). Slides were further 821 processed by deparaffinization (incubation with xylene three times for 3 min, then $822100 \%$ EtOH two times for $3 \mathrm{~min}, 95 \% \mathrm{EtOH}$ two times for $1 \mathrm{~min}, 70 \% \mathrm{EtOH}$ once for 
$8231 \mathrm{~min}$, and $50 \%$ EtOH once for $1 \mathrm{~min}$ ) and rehydration (tap water). Thereafter, antigen

824 retrieval was performed by boiling samples for 20 min in with citrate buffer ( $\mathrm{pH} 6$, for

825 Desmin staining) or Tris-EDTA-Tween buffer ( $\mathrm{pH} \mathrm{9,} \mathrm{for} \mathrm{Myogenin} \mathrm{and} \mathrm{MYOD1).} \mathrm{After}$

826 washing (in TBS-0.025\% Triton, twice $5 \mathrm{~min}$ ) and blocking ( $1.5 \mathrm{~h}$ in TBS-1\%BSA),

827 slides were incubated with primary antibody ( $\alpha$-Desmin 1:400, Abcam ab15200 rabbit

828 antibody; $\alpha-M y o g e n i n$ 1:400, Santa Cruz 5FD mouse antibody; $\alpha-M Y O D 1$ 1:200, Cell

829 Marque EP212 rabbit antibody) overnight at $4{ }^{\circ} \mathrm{C}$. The next day, slides were washed

830 twice with PBS and incubated with secondary antibodies (Desmin and MYOD1: anti-

831 rabbit-HRP, BioRad 170-6515; Myogenin: anti-mouse HRP, BioRad 170-6516) 1:500

832 in PBS-1\% BSA. Stainings were visualized using Liquid DAB+ 2-component system

833 (3,3'-diaminobenzidine, DAKO, Agilent K3467) following the manufacturer's protocol

834 and washed three times with TBS. Counter-staining was performed by incubation with

835 thionine $(0.05 \%$ for $20 \mathrm{~min})$. After subsequent incubation with $96 \% \mathrm{EtOH}, 100 \%$

836 EtOH and xylene, slides were mounted using Permount mounting medium (Fisher

837 Scientific SP15-100) and visualized using a Leica DMi6 microscope.

838 H\&E stainings were performed manually (steps: xylene three times for 5 min, $100 \%$

839 EtOH twice for 1 min, $95 \%$ EtOH twice for $30 \mathrm{sec}, 70 \% \mathrm{EtOH}$ for $30 \mathrm{sec}$, washed in

840 demi-water, incubation with hematoxylin (Hematox 7211) for 2:45 min, washed with

841 demi-water, brief incubations with acidic $\mathrm{EtOH}$, washed with demi-water, $95 \% \mathrm{EtOH}$

842 for $30 \mathrm{sec}$, eosin incubation for 2:45 min, EtOH $70 \%$ for $30 \mathrm{sec}$, EtOH $95 \%$ for $30 \mathrm{sec}$,

$843 \mathrm{EtOH} 100 \%$ twice for $30 \mathrm{sec}$, xylene three times for $1 \mathrm{~min}$ ) or were performed at the

844 in-house pathology department following standard protocols. Visualization was

845 performed as described above.

846 The quality of stainings was evaluated by an in-house pathologist. Representative

847 images of stained spheres are shown and were compared to H\&E as well as IHC 
stainings obtained in pathology for the RMS tumor sample the tumoroid was derived

849 from (representative images chosen by the pathologist).

850

851 RNA and DNA isolation from tumor and derived tumoroid samples for whole-

852 genome sequencing (WGS) and bulk mRNA sequencing (RNA-seq)

853 Tumoroid models:

854 To isolate RNA and DNA for WGS and RNA-seq, tumoroid cells were collected as a pellet, snap frozen and stored at minus $80{ }^{\circ} \mathrm{C}$. Isolation of RNA and DNA from the same pellet was performed using the AllPrep DNA/RNA/miRNA Universal Kit (Qiagen

$857 \quad$ \# 80224) according to the manufacturer's protocol. Lysis in RLT buffer was followed by homogenization using a Qiashredder column as described. The flowthrough fraction in RLT buffer was used for the Allprep DNA and RNA isolations. Quality and quantity of isolated RNA and DNA were measured by using the NanoDrop OneC

861 (Thermo Fisher Scientific), Bioanalyzer 2100 (Agilent), Qubit Fluorometer (Thermo 862 Fisher Scientific), and marker-checks using RT-qPCR (as above).

Tumor samples and germline control samples (EDTA blood):

Resected tissue and/or biopsies were processed within 10 minutes after removal from

866 the patient. DNA and RNA were isolated from the same piece of fresh frozen tissue

867 using the AllPrep DNA/RNA/miRNA Universal Kit (Qiagen \# 80224) using the QIAcube

868 Connect (Qiagen). Reference DNA was isolated from peripheral white blood cells 869 (EDTA blood) using the same method. 
$150 \mathrm{ng}$ of total DNA was used for library preparation using the KAPA HyperPlus kit

874 (Roche), according to manufacturer's instructions. Libraries from tumor and normal tissue were pooled in a 3:1 ratio, with a total of 7 tumor/normal pairs per $\$ 4$ sequencing kit. Libraries were sequenced using 2x150 cycles on a NovaSeq 6000 (Illumina).

Pre-processing, alignment, and annotation:

879 The WGS sequencing data were processed as per the GATK 4.0 best practices 880 workflow for variant calling, using a wdl and cromwell based workflow. Reads were 881 aligned to GRCh38 using bwamem (v0.7.13), and quality control (QC) was performed

882 using FastQC (v0.11.5) and picardTools (v2.20.1). Somatic variants were identified 883 using Mutect2 from GATK v4.1 and annotated using Vep (v92). Likewise, copynumber alterations (CNAs) were identified using GATK v4.1.

887 Raw VCF files from germline, tumor and tumoroid samples were processed with 888 vcftools (v0.1.13) (Danecek et al., 2011). Only variants that met the criteria (passed 889 all quality filters in addition to PHRED quality score 100 (250 for indels) and minimum 890 read depth of 10) were kept for further analyses. Indels were also filtered out if the 891 minimum mapping quality (MQ) was below 60. Bcftools

892 (https://github.com/samtools/bcftools) was used to remove all common snps (VAF $\geq 1$

893 \%) present in dbSNP (https://www.ncbi.nlm.nih.gov/snp/, v151 downloaded in August 894 2020). Filtered files were then loaded into $R(\mathrm{v} 4.0 .2)$ and processed with the package

895 VariantAnnotation (Obenchain et al., 2014) and packages from the tidyverse. Variants 896 in tumor and tumoroid models also present in the germline sample were removed from 897 further analyses; remaining variants were filtered for VAFs $>0.3$ and those tagged as 
missense variant, stop gained, stop lost, start lost, inframe insertion, inframe deletion, and frameshift variant were selected as non-synonymous somatic mutations. Figures were generated with ggplot2 (v.3.3.2).

901

902 Signature analysis:

903 Somatic signature analysis was performed using the $R$ programming language

904 (v3.6.3) and the $R$ packages MutationalPatterns (v3.2.0) and VariantAnnotation variants were filtered on both the variant allele fraction (VAF) and depth (DP). For both

907 the control and the tumor/tumoroid samples we used DP $\geq 20$. For the tumor/tumoroid samples we used VAF $>0.3$, while in control samples the VAF had to be 0 . Samples with 50 or less mutations would have been excluded, but this was not the case for any samples in our cohort. A mutation matrix was generated that shows how often each of

911 the 96 types of base substitutions occurred in each sample. A variational Bayesian

912 non-negative matrix factorization (NMF) was performed on this matrix to extract four

913 de novo mutational signatures for base substitutions. The cosine similarities were then

914 calculated between these de novo signatures and a set of signatures consisting of

915 both the COSMIC signatures (v3.2, GRCh38) and the SIGNAL exposure signatures

916 (Alexandrov et al., 2020; Kucab et al., 2019). The four signatures most similar to the

917 de novo signatures (SBS1, SBS5, SBS18, and Temozolomide..200.uM..1 [TMZ]) were

918 then used for signature refitting. Signature refitting was performed using the

919 fit_to_signatures_strict function using the best_subset method with a max_delta of

920 0.004. Our approach of first performing de novo signature extraction followed by

921 refitting is similar to the approach suggested by Maura et al. (Maura et al., 2019). 
922 To determine the similarity between the samples, the cosine similarities of their base

923 substitution profiles were calculated. This resulted in a cosine similarity matrix that was

924 used to calculate the distance between samples, which was then used for hierarchical

925 clustering.

926

927 Bulk mRNA (RNA-seq) of tumor and tumoroid samples: transcriptional profile and gene fusions

929

Sequencing:

$930300 \mathrm{ng}$ of total RNA was used for library preparation using the KAPA RNA HyperPrep

931 kit with RiboErase (Roche), according to manufacturer's instructions. The protocol was

932 optimized to achieve an insert size of $\sim 300-400 \mathrm{nt}$.

933 RNA libraries were pooled with a total of 25 samples per S1 kit or 60 samples per S2

934 kit. Libraries were sequenced using 2x150 cycles on a NovaSeq 6000 (Illumina).

936 Pre-processing: alignment, annotation, and detection of gene fusions

937 The RNA sequencing data were processed as per the GATK 4.0 best practices 938 workflow for variant calling, using a wdl and cromwell based workflow 939 (https://gatk.broadinstitute.org/hc/en-us/sections/360007226651-Best-Practices-

940 Workflows). This included performing quality control with Fastqc (v0.11.5) to calculate

941 the number of sequencing reads and the insert size (Andrews S., 2010. FastQC: a 942 quality control tool for high throughput sequence data, 943 http://www.bioinformatics.babraham.ac.uk/projects/fastqc). Picard (v2.20.1) for RNA 944 metrics output and MarkDuplicates ("Picard Tools." Broad Institute.

945 http://broadinstitute.github.io/picard/). The raw sequencing reads were aligned using 946 Star (v2.7.0f) to GRCh38 and gencode version 31. Gene fusion detection was 
947 performed using Star fusion (v1.6.0) (Haas et al., 2019). Finally, expression counts

948 were determined at exon and gene level using Subread Counts (Liao et al., 2019).

950 Processing of transcriptome data

951 Raw count tables were loaded into $R(\mathrm{v} 4.0 .2)$ and processed with the packages from

952 the tidyverse. Count matrices were transformed into log2-scale transcripts per million

953 (log2 TPM) tables and genes annotated with the same gene symbol merged.

954 Comparison of transcriptional profiles across samples (kidney and 955 rhabdomyosarcomas) was performed via correlation performed in $R$ using the base 956 package.

957

958 Bulk sequencing data availability

959 The process to make the bulk sequencing data (i.e., whole-genome sequencing and 960 bulk mRNA sequencing) openly available at the European Genome-Phenome Archive 961 (EGA) has been initiated with data upload completed and final curation pending.

962

963 Growth curve experiments

964 Growth behavior in 3D sphere cultures prior to drug screenings (see below) was tested 965 via performing growth curve experiments. Tumoroid cells were plated at different 966 densities (typically between 250 and 4.000 viable cells per well) in 384-well round 967 bottom ultra-low attachment spheroid microplates (Corning, cat no. 3830). To facilitate 968 3D sphere formation, cells were grown in $\mathrm{BM} 1^{*}$ medium without $\mathrm{BME}$ and after 969 dispensing them into the plates, plates were spun (1.500 rpm, $5 \mathrm{~min}$, slow break) to 970 concentrate cells in the center of the wells. Plates were incubated at standard 971 conditions and cells were granted a recovery period of 48 hours. Thereafter, readouts 
were performed using CellTiter-Glo 3D Cell Viability Assay (CTG3D, Promega, cat no. G9683) according to the manufacturer's protocol at three time points: immediately (T0), $48 \mathrm{~h}$ or $72 \mathrm{~h}$ (T48 or T72), and $120 \mathrm{~h}$ (T120). Measured luminescence (via FLUOstar Omega, BMG Labtech) was averaged per readout per cell density and the corresponding background signal from medium was subtracted. Obtained measurements from T48/T72 and T120 were normalized to T0 to calculate the relative growth over the period of $120 \mathrm{~h}$. For the following drug screening experiments (see below), a cell density was chosen that showed logarithmic growth behavior in growth curve experiments. Defined starting number of cells facilitated the establishment of a sphere and were optimized for intrinsic growth factor levels and space depletion in the well over the course of the experiment. Growth curve experiments were performed once per model but with at least ten technical replicates per number of cells plated.

\section{Drug screenings}

986 For drug screenings, tumoroid models were processed according to the same protocol 987 as for the growth curve experiments, with a number of cells seeded that was determined in those experiments (see above). Drugs, dissolved in DMSA or water, were added 48 hours after seeding of the tumoroid spheres fully automated via a robotics system: Up until 2019, this was facilitated via a Caliper Sciclone-Robotic

991 Liquid Handler using a dilution of the drugs in medium and transferring this dilution to 992 the cells by pipetting. From 2020 onwards, screenings were performed at the highthroughput screening (HTS) facility of the Princess Máxima Center with a Biomek i7

994 liquid handler, using the acoustic liquid handler Echo550 for direct drug transfers. 995 Before the screening, the 384-well working plates containing the dissolved drugs are 996 shaken (30 min, RT) and centrifuged (1 min, $1500 \mathrm{rpm}$ ). Tumoroid spheres were 
treated with a ten-fold dilution series of the drugs on the library plate $(0.1 \mathrm{nM}$ to 10 $\mu \mathrm{M})$. Positive control samples were treated with DMSO, negative control samples with staurosporine (final concentration of $10 \mu \mathrm{M}$ ). Readouts were performed using

1000 CellTiter-Glo 3D Cell Viability Assay (CTG3D, Promega, cat no. G9683) according to

1001 the manufacturer's protocol at T0 (before addition of drugs - control) and at T120 (120

$1002 \mathrm{~h}$ after addition of drugs - readout). Dose-response was estimated per drug and concentration in relation to the DMSO-treated cells (set to 100\%) and empty controls (set to $0 \%$ ). Quality of the screenings was approved after assessment of the cell growth (absorbance signal of T120 over T0), the negative, positive, and empty controls and, if applicable, the amount of variability between the duplicates.

1007 Depending on the available number of cells at drug screening, a subset of models 1008 (RMS007, RMS109, RMS110, RMS000EEC, RMS000ETY, RMS000FLV, 1009 RMS000HQC, RMS000HWO, RMS000HWQ) could be screened in technical 1010 duplicates while for the other models (RMS006, RMS012, RMS013, RMS102, 1011 RMS108, RMS127, RMS335, RMS410, RMS444, RMS000CPU) only screening 1012 without technical duplicates was feasible. To test reproducibility, we analyzed the 1013 correlation between the technical duplicates in the tumoroid models for which those 1014 were available (Suppl. Fig. 5). As we observed a very high correlation there $(R=0.91)$, 1015 indicating a high reproducibility, we deemed it appropriate to combine the analysis of 1016 samples tested in duplicate and those not tested in duplicate given the scope of the 1017 assay was to assess whether we could obtain biologically meaningful results (any "hit" 1018 should have been further validated). RMS tumoroid models RMSO00ETY and 1019 RMS000HWO were excluded from the analysis as they did not show an increase of 1020 CTG3D signal between T0 and T120 (Suppl. Table 2), indicating that these two models 
did not grow sufficiently under the screening conditions (while they displayed a minor

1022 increase in signal during the growth curve experiments).

1023 Further data analysis was performed using $R$ (v3.6.3). Area Under the Curve (AUC)

1024 values were calculated for every drug per tumoroid model and replicate (in the case

1025 of the tumoroid models with technical replicates) using the auc function of the MESS

1026 package (v0.5.6). In the case of RMS tumoroid models with technical duplicates, the

1027 two resulting AUC values per drug were correlated (using the cor function of base $R$ )

1028 to calculate the correlation between replicates and plotted using the plot function of

1029 base $R$ (see above). For the further downstream analysis, these replicate AUC values

1030 were averaged, resulting in a matrix with one AUC value per drug per RMS tumoroid model. On this matrix, unsupervised clustering was performed using the get_dist

1032 function from the factoextra package (v1.0.7) using the arguments "pearson" for RMS

1033 tumoroid models and "euclidean" for drugs to measure dissimilarity with further

1034 clustering using the hclust function from base $R$ using the "average" argument for

1035 linkage. Data were visualized using the heatmap.2 function from the gplots package

1036 (v3.0.3) using dendrograms generated from the established clustering. Principal component analysis (PCA) was performed using the prcomp function from base $R$ with exclusion of the RMS tumoroid model RMS000FLV due to its outlier behavior. PCAs were visualized using the fviz_pca_ind and fviz_pca_var functions from the above-

1040 mentioned factoextra package.

\section{CRISPR/Cas9 knockout of TP53 and functional evaluation}

1043 RMS012 tumoroid cells were kept under standard conditions (BM1* supplemented

1044 with $0.1 \%$ BME) prior to the experiment. Upon reaching $70 \%$ confluency, tumoroid 1045 cells were passaged as usual and seeded with high density into wells of a 24-well 
1046

1047

1048

1049

1050

1051

1052

1053

1054

1055

1056

1057

1058

1059

1060

1061

1062

1063

1064

1065

1066

1067

1068

1069

1070

plate. After $24 \mathrm{~h}$ of recovery, transfection was performed: Nucleic acid-Lipofectamine 2000 complexes were prepared according to the standard Lipofectamine 2000 protocol (Invitrogen). Four $\mu \mathrm{l}$ of Lipofectamine 2000 reagent in $50 \mu \mathrm{l}$ Opti-MEM medium (Gibco) and $1.5 \mu \mathrm{g}$ of DNA (pSpCas9(BB)-2A-GFP control or sgRNA TP53 plasmid in $50 \mu \mathrm{l}$ Opti-MEM medium) were mixed, incubated for $5 \mathrm{~min}$, and added to the cells. Plasmids were kindly shared by Jarno Drost (PMC, NL). For the plasmid sequence refer to Drost et al. 2015 (Drost et al., 2015). Twenty-four hours after transfection, transfection efficiency was evaluated using fluorescence microscopy, detecting GFP positive cells. Forty-eight hours after transfection, selection with nutlin$3(10 \mu \mathrm{M})$ was started. Four days after the start of selection, first nutlin-3 resistant colonies could be detected in TP53 knockout plasmid transfected cells whereas cells transfected with control plasmids died due to nutlin-3 exposure. Putative knockout cells were further expanded.

Knockout was confirmed using Western Blotting and Sanger sequencing of genomic DNA: For Western Blotting, snap frozen tumoroid cell pellets were lysed in Phosphatase-substituted RIPA buffer and run on a $10 \%$ precast gel for P53 detection (BioRad). Protein levels of P53 (1:1.000, Santa Cruz DO-1 P53 antibody) were detected while Histone 3 (1:2.000, Abcam ab1791 Pan-H3) served as loading control. For visualization, secondary antibodies (goat anti-rabbit, BioRad 1706515 / goat antimouse BioRad 1706516) conjugated with horseradish peroxidase (HRP) were used together with ECL substrate (Perkin Elmer) on an imaginer (BioRad ChemiDoc). Western Blotting analysis was performed twice, and a representative blot is shown. For Sanger sequencing of genomic DNA, a snap frozen tumoroid cell pellet was lysed using DirectPCR Lysis Reagent for Cells (Viagen) according to the manufacturer's protocol. PCR amplification was performed using Phusion High-Fidelity DNA 
1071 Polymerase (New England BioLabs) using the following primers: forward 5'-

1072 CCCATCTACAGTCCCCCTTG-3', reverse 5'-CAGGAAGCCAAAGGGTGAAGA-3'.

1073 PCR products were cleaned up and concentrated using a DNA Clean-up and

1074 Concentration Kit (Zymo Research) according to the manufacturer's protocol. Gel

1075 electrophoresis indicated the presence of a specific product which was sent for Sanger sequencing using the following primers: forward 5'-TGGTTCACTGAAGACCCAGG-3', reverse 5'-GAAGTCTCATGGAAGCCAGCC-3'. Obtained sequences were aligned and inspected using the Benchling browser tool (https://www.benchling.com/). Furthermore, sequencing data was submitted for TIDE (Tracking of Indels by

1080 Decomposition, http://tide.nki.nl) analysis to infer the composition of Indels in the 1081 knockout population (Brinkman et al., 2014).

1082 For detection of DNA double strand breaks, induction of $\mathrm{yH} 2 \mathrm{AX}$ was measured using 1083 Western Blotting. Fusion-negative embryonal rhabdomyosarcoma cell line RD (kindly 1084 shared by Jan Molenaar, PMC, NL) served as a control (either untreated or treated 1085 with $1 \mu \mathrm{M}, 5 \mu \mathrm{M}$, or $10 \mu \mathrm{M}$ of staurosporine (Sigma) for $24 \mathrm{~h}$ ). RD cells were cultured 1086 under conventional conditions as detailed by the American Type Culture Collection 1087 (ATCC) with regular testing for mycoplasma contamination. For Western Blotting, snap 1088 frozen tumoroid (RMS012 TP53 KO) or tumor (RD) cell pellets were lysed in 1089 Phosphatase-substituted RIPA buffer and run on a $15 \%$ self-cast gel for $\mathrm{yH} 2 \mathrm{AX}$ 1090 (p.S139) detection. Protein levels of $\mathrm{yH} 2 \mathrm{AX}$ (p.S139, 1:2.000, Abcam ab26350 antibody) were detected while GAPDH (1:1.000, Abcam ab9485 antibody) served as loading control. Visualization was performed as described above for the confirmation

1093 of the knockout. As above, Western Blotting analysis was performed twice, and a 1094 representative blot is shown. 
1095 To assess differential response to prexasertib (MedchemExpress), RMS012 tumoroid

1096 models (TP53 wildtype and TP53 knockout) were processed according to the same

1097 protocol as for the growth curve experiments (see above), with 500 cells seeded per

1098 well. Prexasertib (dilution series from $200 \mathrm{nM}$ to $0.78125 \mathrm{nM}$ ) was added 48 hours

1099 after seeding of the tumoroid spheres. Control samples were treated with DMSO.

1100 Readouts were performed using CellTiter-Glo 3D Cell Viability Assay (CTG3D,

1101 Promega, cat no. G9683) according to the manufacturer's protocol at T72 (72 $\mathrm{h}$ after

1102 addition of drugs). Dose-response was estimated per model and concentration in

1103 relation to the DMSO-treated cells (set to 100\%). The experiment was performed three

1104 times. Further data analysis was performed using $R$ (v3.6.3). A sigmoidal fit for the

1105 dose-response curve was calculated per replicate for both the knockout and wildtype

1106 sample with a three-parameter log-logistics function using the drc package (v3.0-1)

1107 (Ritz et al., 2015). The statistical significance of the differences in fitted $I_{50}$ values

1108 between knockout and wildtype were obtained using a two-sided t-test. For

1109 visualization purposes a three-parameter sigmoidal fit per model (not per replicate)

1110 was used.

1111

1112 Image post-processing and figure preparation

1113 Microscopy images from RMS tumoroid IHC and H\&E stainings as well as images

1114 from Western Blotting were post-processed according to good scientific practice with

1115 Adobe Photoshop 2021 and Fiji (v2.0.0-rc-69/1.52i) (Schindelin et al., 2012). Images

1116 from original RMS tumors (H\&E and IHC) were not processed. Figures were prepared

1117 using Adobe Illustrator 2021.

1118

1119 Code availability 
bioRxiv preprint doi: https://doi.org/10.1101/2022.01.03.474504; this version posted January 4,2022 . The copyright holder for this preprint

(which was not certified by peer review) is the author/funder, who has granted bioRxiv a license to display the preprint in perpetuity. It is made available under aCC-BY-NC 4.0 International license.

1120 Code is made openly available on https://github.com/teresouza/rms2018-009.

1121 


\section{Supplemental items legends}

\section{Figure legends}

1125 Suppl. Fig. 1: Morphological (via H\&E) and immunohistochemical (IHC) comparison

1126 of RMS tumors and derived RMS tumoroid models shows retained marker protein

1127 (Desmin, Myogenin, and MYOD1) expression and cellular morphology. Scale bars 1128 equal $200 \mu \mathrm{m}$.

1130 Suppl. Fig. 2: (A) Examples of circular copy number plots of fusion-negative RMS 1131 with embryonal (RMS000ETY) or alveolar (RMS007) histology. Outer circle depicts

1132 the tumoroid model, inner circle depicts the tumor. (B) Examples of circular copy 1133 number plots of fusion-positive RMS with alveolar histology and PAX3-FOXO1 fusion

1134 (RMS410) or PAX7-FOXO1 fusion (RMS000HQC). Outer circle depicts the tumoroid 1135 model, inner circle depicts the tumor. (C) Contribution of averaged single base 1136 substitution (SBS) profiles for RMS tumors (upper row) and tumoroid models (lower

1137 row). (D) Clustered correlogram of SBS profiles detected in RMS tumors (T) and 1138 tumoroid models (O).

1140 Suppl. Fig. 3: (A) Circular copy number plots of standard and late passage RMS 1141 tumoroid models of a fusion-negative alveolar (RMS007) and a PAX7-FOXO1 positive 1142 alveolar (RMS335) RMS. Outer circle depicts the standard passage tumoroid, inner 1143 circle depicts the late passage tumoroid model. (B) Circular copy number plots of

1144 standard passage and independently derived RMS tumoroid models of two fusion1145 negative embryonal RMS (RMS012 and RMS444). Outer circle depicts the standard 1146 passage tumoroid, inner circle depicts the independently derived tumoroid model. (C) 
1147 Contribution of averaged single base substitution (SBS) profiles for standard (O),

1148 independently derived (O2), and late passage (OL) RMS tumoroid models. (D)

1149 Clustered correlogram of SBS profiles detected in standard (O), independently derived

1150 (O2), and late passage (OL) RMS tumoroid models.

1151

1152 Suppl. Fig. 4: (A) Left panel: Inferred Indel Spectrum from Sanger sequencing using

1153 the TIDE tool (https://tide.nki.nl). Right panel: Sanger sequencing around Cas9 cut site

1154 (arrow at the bottom) in TP53 gene. Protospacer Adjacent Motif (PAM) sequence

1155 indicated. (B) Western Blotting analysis of $\mathrm{yH} 2 \mathrm{AX}$ (Ser-139) as proxy for DNA double-

1156 strand breaks in untreated RMS012 tumoroid cells (TP53 wildtype and TP53

1157 knockout). RMS cell line RD treated with staurosporine served as positive control while

1158 GAPDH served as loading control.

1160 Suppl. Fig. 5: Correlation analysis of Area Under the Curve (AUC) values from 1161 technical replicates of drug screenings of RMS007, RMS109, RMS110, RMS000EEC,

1162 RMS000FLV, RMS000HQC, and RMS000HWQ. Overall, the correlation coefficient $1163(R)$ is 0.91.

1164

1165 Table legends

1166 Suppl. Table 1: Clinical annotation RMS tumoroid model cohort.

1168 Suppl. Table 2: Relative growth of vehicle treated control tumoroid cells during drug screenings. 


\section{References}

1173

1174 Abdullah, K.G., Bird, C.E., Buehler, J.D., Gattie, L.C., Savani, M.R., Sternisha, A.C.,

1175 Xiao, Y., Levitt, M.M., Hicks, W.H., Li, W., et al. (2021). Establishment of patient-

1176 derived organoid models of lower grade glioma. Neuro. Oncol.

1177 Acanda De La Rocha, A.M., Fader, M., Coats, E.R., Espinal, P.S., Berrios, V.,

1178 Saghira, C., Sotto, I., Shakya, R., Janvier, M., Khatib, Z., et al. (2021). Clinical Utility

1179 of Functional Precision Medicine in the Management of Recurrent/Relapsed

1180 Childhood Rhabdomyosarcoma. JCO Precis. Oncol. 1659-1665.

1181 Alexandrov, L.B., Kim, J., Haradhvala, N.J., Huang, M.N., Tian Ng, A.W., Wu, Y.,

1182 Boot, A., Covington, K.R., Gordenin, D.A., Bergstrom, E.N., et al. (2020). The

1183 repertoire of mutational signatures in human cancer. Nature 578, 94-101.

1184 Amary, M.F.C., Berisha, F., Bernardi, F.D.C., Herbert, A., James, M., Reis-Filho,

1185 J.S., Fisher, C., Nicholson, A.G., Tirabosco, R., Diss, T.C., et al. (2007). Detection of

1186 SS18-SSX fusion transcripts in formalin-fixed paraffin-embedded neoplasms:

1187 analysis of conventional RT-PCR, qRT-PCR and dual color FISH as diagnostic tools

1188 for synovial sarcoma. Mod. Pathol. 2007204 20, 482-496.

1189 Belyea, B.C., Naini, S., Bentley, R.C., and Linardic, C.M. (2011). Inhibition of the

1190 notch-hey1 axis blocks embryonal rhabdomyosarcoma tumorigenesis. Clin. Cancer

1191 Res. 17, 7324-7336.

1192 Bersani, F., Taulli, R., Accornero, P., Morotti, A., Miretti, S., Crepaldi, T., and

1193 Ponzetto, C. (2008). Bortezomib-mediated proteasome inhibition as a potential

1194 strategy for the treatment of rhabdomyosarcoma. Eur. J. Cancer 44, 876-884.

1195 Bisogno, G., De Salvo, G.L., Bergeron, C., Gallego Melcón, S., Merks, J.H., Kelsey,

1196 A., Martelli, H., Minard-Colin, V., Orbach, D., Glosli, H., et al. (2019). Vinorelbine and 
continuous low-dose cyclophosphamide as maintenance chemotherapy in patients

1200 Bleijs, M., Wetering, M., Clevers, H., and Drost, J. (2019). Xenograft and organoid 1201 model systems in cancer research. EMBO J. 38, e101654.

1202 Blokzijl, F., Janssen, R., van Boxtel, R., and Cuppen, E. (2018). MutationalPatterns:

1203 Comprehensive genome-wide analysis of mutational processes. Genome Med. 10.

1204 Boulay, G., Cironi, L., Garcia, S.P., Rengarajan, S., Xing, Y.H., Lee, L., Awad, M.E., 1205 Naigles, B., lyer, S., Broye, L.C., et al. (2021). The chromatin landscape of primary 1206 synovial sarcoma organoids is linked to specific epigenetic mechanisms and 1207 dependencies. Life Sci. Alliance 4.

1208 Brinkman, E.K., Chen, T., Amendola, M., and Van Steensel, B. (2014). Easy

1209 quantitative assessment of genome editing by sequence trace decomposition.

$1210 \quad$ Nucleic Acids Res. 42, e168-e168.

1211 Brodin, B.A., Wennerberg, K., Lidbrink, E., Brosjö, O., Potdar, S., Wilson, J.N., Ma,

1212 L., Moens, L.N., Hesla, A., Porovic, E., et al. (2019). Drug sensitivity testing on

1213 patient-derived sarcoma cells predicts patient response to treatment and identifies c-

1214 Sarc inhibitors as active drugs for translocation sarcomas. Br. J. Cancer 120, 435-

\section{3.}

1216 Burningham, Z., Hashibe, M., Spector, L., and Schiffman, J.D. (2012). The

1217 Epidemiology of Sarcoma. Clin. Sarcoma Res. 2, 14.

1218 Calandrini, C., Schutgens, F., Oka, R., Margaritis, T., Candelli, T., Mathijsen, L.,

1219 Ammerlaan, C., van Ineveld, R.L., Derakhshan, S., de Haan, S., et al. (2020). An

1220 organoid biobank for childhood kidney cancers that captures disease and tissue

1221 heterogeneity. Nat. Commun. 11. 
1222 Clevers, H. (2016). Modeling Development and Disease with Organoids. Cell 165,

1223 1586-1597.

1224 Custers, L., Khabirova, E., Coorens, T.H.H., Oliver, T.R.W., Calandrini, C., Young,

1225 M.D., Vieira Braga, F.A., Ellis, P., Mamanova, L., Segers, H., et al. (2021). Somatic

1226 mutations and single-cell transcriptomes reveal the root of malignant rhabdoid

1227 tumours. Nat. Commun. 12, 1-11.

1228 Danecek, P., Auton, A., Abecasis, G., Albers, C.A., Banks, E., DePristo, M.A.,

1229 Handsaker, R.E., Lunter, G., Marth, G.T., Sherry, S.T., et al. (2011). The variant call

1230 format and VCFtools. Bioinformatics 27, 2156-2158.

1231 Defachelles, A.-S., Bogart, E., Casanova, M., Merks, J.H.M., Bisogno, G., Calareso,

1232 G., Gallego Melcon, S., Gatz, S.A., Le Deley, M.-C., McHugh, K., et al. (2021).

1233 Randomized Phase II Trial of Vincristine-Irinotecan With or Without Temozolomide,

1234 in Children and Adults With Relapsed or Refractory Rhabdomyosarcoma: A

1235 European Paediatric Soft Tissue Sarcoma Study Group and Innovative Therapies for

1236 Children With Cancer . J. Clin. Oncol. 39, 2979-2990.

1237 Dias, P., Chen, B., Dilday, B., Palmer, H., Hosoi, H., Singh, S., Wu, C., Li, X.,

1238 Thompson, J., Parham, D., et al. (2000). Strong immunostaining for myogenin in

1239 rhabdomyosarcoma is significantly associated with tumors of the alveolar subclass.

1240 Am. J. Pathol. 156, 399-408.

1241 Dijkstra, K.K., Monkhorst, K., Schipper, L.J., Hartemink, K.J., Smit, E.F., Kaing, S.,

1242 de Groot, R., Wolkers, M.C., Clevers, H., Cuppen, E., et al. (2020). Challenges in

1243 Establishing Pure Lung Cancer Organoids Limit Their Utility for Personalized

1244 Medicine. Cell Rep. 31.

1245 Dobbelstein, M., and Sørensen, C.S. (2015). Exploiting replicative stress to treat

1246 cancer. Nat. Rev. Drug Discov. 14, 405-423. 
1247 Drost, J., Van Jaarsveld, R.H., Ponsioen, B., Zimberlin, C., Van Boxtel, R., Buijs, A.,

1248 Sachs, N., Overmeer, R.M., Offerhaus, G.J., Begthel, H., et al. (2015). Sequential

1249 cancer mutations in cultured human intestinal stem cells. Nature $521,43-47$.

1250 Drummond, C.J., Hanna, J.A., Garcia, M.R., Devine, D.J., Heyrana, A.J., Finkelstein,

1251 D., Rehg, J.E., and Hatley, M.E. (2018). Hedgehog Pathway Drives Fusion-Negative

1252 Rhabdomyosarcoma Initiated From Non-myogenic Endothelial Progenitors. Cancer

1253 Cell 33, 108-124.e5.

1254 Enterline, H.T., and Horn, R.C. (1958). Alveolar rhabdomyosarcoma; a distinctive

1255 tumor type. Am. J. Clin. Pathol. 29, 356-366.

1256 Felix, C.A., Winick, N.J., Crouch, G.D., and Helman, L.J. (1992). Frequency and

1257 Diversity of p53 Mutations in Childhood Rhabdomyosarcoma. Cancer Res. 52,

$1258 \quad 2243-2247$.

1259 Fusco, P., Parisatto, B., Rampazzo, E., Persano, L., Frasson, C., Di Meglio, A.,

1260 Leslz, A., Santoro, L., Cafferata, B., Zin, A., et al. (2019). Patient-derived organoids

1261 (PDOs) as a novel in vitro model for neuroblastoma tumours. BMC Cancer 19, 1-11.

1262 Gao, H., Korn, J.M., Ferretti, S., Monahan, J.E., Wang, Y., Singh, M., Zhang, C.,

1263 Schnell, C., Yang, G., Zhang, Y., et al. (2015). High-throughput screening using

1264 patient-derived tumor xenografts to predict clinical trial drug response. Nat. Med. 21,

1265 1318-1325.

1266 Glosli, H., Bisogno, G., Kelsey, A., Chisholm, J.C., Gaze, M., Kolb, F., McHugh, K.,

1267 Shipley, J., Gallego, S., Merks, J.H.M., et al. (2021). Non-parameningeal head and

1268 neck rhabdomyosarcoma in children, adolescents, and young adults: Experience of

1269 the European paediatric Soft tissue sarcoma Study Group (EpSSG) - RMS2005

1270 study. Eur. J. Cancer 151, 84-93.

1271 Haas, B.J., Dobin, A., Li, B., Stransky, N., Pochet, N., and Regev, A. (2019). 
1272 Accuracy assessment of fusion transcript detection via read-mapping and de novo

1273 fusion transcript assembly-based methods. Genome Biol. 20.

1274 Heerema-Mckenney, A., Wijnaendts, L.C.D., Pulliam, J.F., Lopez-Terrada, D.,

1275 McKenney, J.K., Zhu, S., Montgomery, K., Mitchell, J., Marinelli, R.J., Hart, A.A.M.,

1276 et al. (2008). Diffuse myogenin expression by immunohistochemistry is an

1277 independent marker of poor survival in pediatric rhabdomyosarcoma: A tissue

1278 microarray study of 71 primary tumors including correlation with molecular

1279 phenotype. Am. J. Surg. Pathol. 32, 1513-1522.

1280 Hettmer, S., and Wagers, A.J. (2010). Muscling in: Uncovering the origins of

1281 rhabdomyosarcoma. Nat. Med. 16, 171-173.

1282 Hinson, A.R.P., Jones, R., Lisa, L.E., Belyea, B.C., Barr, F.G., and Linardic, C.M.

1283 (2013). Human rhabdomyosarcoma cell lines for rhabdomyosarcoma research:

1284 Utility and pitfalls. Front. Oncol. 3 JUL.

1285 Imle, R., Kommoss, F.K.F., and Banito, A. (2021). Preclinical In Vivo Modeling of

1286 Pediatric Sarcoma-Promises and Limitations. J. Clin. Med. 10, 1578.

1287 Jacob, F., Salinas, R.D., Zhang, D.Y., Nguyen, P.T.T., Schnoll, J.G., Wong, S.Z.H.,

1288 Thokala, R., Sheikh, S., Saxena, D., Prokop, S., et al. (2020). A Patient-Derived

1289 Glioblastoma Organoid Model and Biobank Recapitulates Inter- and Intra-tumoral

1290 Heterogeneity. Cell 180, 188-204.e22.

1291 Jinek, M., Chylinski, K., Fonfara, I., Hauer, M., Doudna, J.A., and Charpentier, E.

1292 (2012). A programmable dual-RNA-guided DNA endonuclease in adaptive bacterial 1293 immunity. Science (80-. ). 337, 816-821.

1294 Kashi, V.P., Hatley, M.E., and Galindo, R.L. (2015). Probing for a deeper

1295 understanding of rhabdomyosarcoma: insights from complementary model systems.

1296 Nat Rev Cancer 15, 426-439. 
1297 Kersten, K., Visser, K.E., Miltenburg, M.H., and Jonkers, J. (2017). Genetically

1298 engineered mouse models in oncology research and cancer medicine. EMBO Mol.

1299 Med. 9, 137-153.

1300 Kim, J., Koo, B.K., and Knoblich, J.A. (2020). Human organoids: model systems for 1301 human biology and medicine. Nat. Rev. Mol. Cell Biol. 21, 571-584.

1302 Kucab, J.E., Zou, X., Morganella, S., Joel, M., Nanda, A.S., Nagy, E., Gomez, C.,

1303 Degasperi, A., Harris, R., Jackson, S.P., et al. (2019). A Compendium of Mutational

1304 Signatures of Environmental Agents. Cell 177, 821-836.e16.

1305 Lee, J.M., Nair, J., Zimmer, A., Lipkowitz, S., Annunziata, C.M., Merino, M.J.,

1306 Swisher, E.M., Harrell, M.I., Trepel, J.B., Lee, M.J., et al. (2018). Prexasertib, a cell

1307 cycle checkpoint kinase 1 and 2 inhibitor, in BRCA wild-type recurrent high-grade

1308 serous ovarian cancer: a first-in-class proof-of-concept phase 2 study. Lancet Oncol.

1309 19, 207-215.

1310 Li, J., Thompson, T.D., Miller, J.W., Pollack, L.A., and Stewart, S.L. (2008). Cancer

1311 incidence among children and adolescents in the United States, 2001-2003.

1312 Pediatrics 121.

1313 Liao, Y., Smyth, G.K., and Shi, W. (2019). The R package Rsubread is easier, faster,

1314 cheaper and better for alignment and quantification of RNA sequencing reads.

1315 Nucleic Acids Res. 47, e47-e47.

1316 Loarer, F. Le, Laffont, S., Lesluyes, T., Tirode, F., Antonescu, C., Baglin, A.C.,

1317 Delespaul, L., Soubeyran, I., Hostein, I., Pérot, G., et al. (2019). Clinicopathologic

1318 and Molecular Features of a Series of 41 Biphenotypic Sinonasal Sarcomas

1319 Expanding Their Molecular Spectrum. Am. J. Surg. Pathol. 43, 747-754.

1320 Lu, J., Krepelova, A., Rasa, S.M.M., Annunziata, F., Husak, O., Adam, L., Nunna, S., 1321 and Neri, F. (2021). Characterization of an in vitro 3D intestinal organoid model by 
using massive RNAseq-based transcriptome profiling. Sci. Rep. 11, 1-14.

1323 Ma, C.X., Cai, S., Li, S., Ryan, C.E., Guo, Z., Schaiff, W.T., Lin, L., Hoog, J., Goiffon,

1324 R.J., Prat, A., et al. (2012). Targeting Chk1 in p53-deficient triple-negative breast

1325 cancer is therapeutically beneficial in human-in-mouse tumor models. J. Clin. Invest.

1326 122, 1541-1552.

1327 Manders, F., Brandsma, A.M., Kanter, J. de, Verheul, M., Oka, R., Roosmalen, M.J.

1328 van, Roest, B. van der, Hoeck, A. van, Cuppen, E., and Boxtel, R. van (2021).

1329 MutationalPatterns: The one stop shop for the analysis of mutational processes.

$1330 \quad$ BioRxiv 2021.11.01.466730.

1331 Manzella, G., Schreck, L.D., Breunis, W.B., Molenaar, J., Merks, H., Barr, F.G., Sun,

1332 W., Römmele, M., Zhang, L., Tchinda, J., et al. (2020). Phenotypic profiling with a

1333 living biobank of primary rhabdomyosarcoma unravels disease heterogeneity and

1334 AKT sensitivity. Nat. Commun. 11, 1-15.

1335 Mascarenhas, L., Lyden, E.R., Breitfeld, P.P., Walterhouse, D.O., Donaldson, S.S.,

1336 Rodeberg, D.A., Parham, D.M., Anderson, J.R., Meyer, W.H., and Hawkins, D.S.

1337 (2019). Risk-based treatment for patients with first relapse or progression of

1338 rhabdomyosarcoma: A report from the Children's Oncology Group. Cancer 125,

1339 2602-2609.

1340 Maura, F., Degasperi, A., Nadeu, F., Leongamornlert, D., Davies, H., Moore, L.,

1341 Royo, R., Ziccheddu, B., Puente, X.S., Avet-Loiseau, H., et al. (2019). A practical

1342 guide for mutational signature analysis in hematological malignancies. Nat.

1343 Commun. 10.

1344 Obenchain, V., Lawrence, M., Carey, V., Gogarten, S., Shannon, P., and Morgan, M.

1345 (2014). VariantAnnotation: A Bioconductor package for exploration and annotation of 1346 genetic variants. Bioinformatics 30, 2076-2078. 
1347 Pappo, A.S., Anderson, J.R., Crist, W.M., Wharam, M.D., Breitfeld, P.P., Hawkins,

1348 D., Raney, R.B., Womer, R.B., Parham, D.M., Qualman, S.J., et al. (1999). Survival

1349 after relapse in children and adolescents with rhabdomyosarcoma: A report from the

1350 intergroup rhabdomyosarcoma study group. J. Clin. Oncol. 17, 3487-3493.

1351 Patton, R.B., and Horn, R.G. (1962). Rhabdomyosarcoma: Clinical and pathological

1352 features and comparison with human fetal and embryonal skeletal muscle. Surgery

$1353 \quad 52,572-584$.

1354 Perez, E.A., Kassira, N., Cheung, M.C., Koniaris, L.G., Neville, H.L., and Sola, J.E.

1355 (2011). Rhabdomyosarcoma in children: A SEER population based study. J. Surg.

1356 Res. 170, e243-e251.

1357 Petljak, M., Alexandrov, L.B., Brammeld, J.S., Price, S., Wedge, D.C., Grossmann,

1358 S., Dawson, K.J., Ju, Y.S., Iorio, F., Tubio, J.M.C., et al. (2019). Characterizing

1359 Mutational Signatures in Human Cancer Cell Lines Reveals Episodic APOBEC

1360 Mutagenesis. Cell 176, 1282-1294.e20.

1361 Pompili, L., Porru, M., Caruso, C., Biroccio, A., and Leonetti, C. (2016). Patient-

1362 derived xenografts: A relevant preclinical model for drug development. J. Exp. Clin.

1363 Cancer Res. 35.

1364 Ponce-Castañeda, M.V., García-Chéquer, A.J., Eguía Aguilar, P., Abundes-Ramírez,

1365 M.A., Hernández-Angeles, A., Nieto-Martínez, K., Gómez-Laguna, L., Sadowinski-

1366 Pine, S., and Cabrera-Muñoz, M. de L. (2014). Detection of common chromosomal

1367 translocations in small round blue cell pediatric tumors. Arch. Med. Res. 45, 143-

1368151.

1369 Ritz, C., Baty, F., Streibig, J.C., and Gerhard, D. (2015). Dose-response analysis

1370 using R. PLoS One 10.

1371 Rogakou, E.P., Pilch, D.R., Orr, A.H., Ivanova, V.S., and Bonner, W.M. (1998). DNA 
double-stranded breaks induce histone H2AX phosphorylation on serine 139. J. Biol.

Chem. 273, 5858-5868.

1374 Rudzinski, E.R., Anderson, J.R., Hawkins, D.S., Skapek, S.X., Parham, D.M., and

1375 Teot, L.A. (2015). The world health organization classification of skeletal muscle

1376 tumors in pediatric rhabdomyosarcoma a report from the children's oncology group.

1377 Arch. Pathol. Lab. Med. 139, 1281-1287.

1378 Saltsman, J.A., Hammond, W.J., Narayan, N.J.C., Requena, D., Gehart, H., Lalazar,

1379 G., Laquaglia, M.P., Clevers, H., and Simon, S. (2020). A human organoid model of 1380 aggressive hepatoblastoma for disease modeling and drug testing. Cancers (Basel).

$1381 \quad 12,1-18$.

1382 Sato, T., Vries, R.G., Snippert, H.J., Van De Wetering, M., Barker, N., Stange, D.E.,

1383 Van Es, J.H., Abo, A., Kujala, P., Peters, P.J., et al. (2009). Single Lgr5 stem cells

1384 build crypt-villus structures in vitro without a mesenchymal niche. Nature 459, 262-

1385265.

1386 Sato, T., Stange, D.E., Ferrante, M., Vries, R.G.J., Van Es, J.H., Van Den Brink, S.,

1387 Van Houdt, W.J., Pronk, A., Van Gorp, J., Siersema, P.D., et al. (2011). Long-term expansion of epithelial organoids from human colon, adenoma, adenocarcinoma, and Barrett's epithelium. Gastroenterology 141, 1762-1772.

1390 Schindelin, J., Arganda-Carreras, I., Frise, E., Kaynig, V., Longair, M., Pietzsch, T.,

1391 Preibisch, S., Rueden, C., Saalfeld, S., Schmid, B., et al. (2012). Fiji: An open-

1392 source platform for biological-image analysis. Nat. Methods 9, 676-682.

1393 Shern, J.F., Chen, L., Chmielecki, J., Wei, J.S., Patidar, R., Rosenberg, M.,

1394 Ambrogio, L., Auclair, D., Wang, J., Song, Y.K., et al. (2014). Comprehensive

1395 genomic analysis of rhabdomyosarcoma reveals a landscape of alterations affecting 1396 a common genetic axis in fusion-positive and fusion-negative tumors. Cancer Discov 
1398 Shern, J.F., Selfe, J., Izquierdo, E., Patidar, R., Chou, H.-C., Song, Y.K., Yohe, M.E.,

1399 Sindiri, S., Wei, J., Wen, X., et al. (2021). Genomic Classification and Clinical

1400 Outcome in Rhabdomyosarcoma: A Report From an International Consortium. J.

1401 Clin. Oncol. 39, 2859-2871.

1402 Skapek, S.X., Ferrari, A., Gupta, A.A., Lupo, P.J., Butler, E., Shipley, J., Barr, F.G., and Hawkins, D.S. (2019). Rhabdomyosarcoma. Nat Rev Dis Prim. 5, 1.

1404 Stewart, E., Federico, S.M., Chen, X., Shelat, A.A., Bradley, C., Gordon, B.,

1405 Karlstrom, A., Twarog, N.R., Clay, M.R., Bahrami, A., et al. (2017). Orthotopic

1406 patient-derived xenografts of paediatric solid tumours. Nature 549, 96-100.

1407 Wachtel, M., Dettling, M., Koscielniak, E., Stegmaier, S., Treuner, J., Simon-

1408 Klingenstein, K., Bühlmann, P., Niggli, F.K., and Schäfer, B.W. (2004). Gene expression signatures identify rhabdomyosarcoma subtypes and detect a novel $\mathrm{t}(2 ; 2)(\mathrm{q} 35 ; \mathrm{p} 23)$ translocation fusing PAX3 to NCOA1. Cancer Res. 64, 5539-5545.

1411 Weber-Hall, S., Anderson, J., McManus, A., Abe, S., Nojima, T., Pinkerton, R.,

1412 Pritchard-Jones, K., and Shipley, J. (1996). Gains, losses, and amplification of

1413 genomic material in rhabdomyosarcoma analyzed by comparative genomic

1414 hybridization. Cancer Res. 56, 3220-3224.

1415 Van De Wetering, M., Francies, H.E., Francis, J.M., Bounova, G., lorio, F., Pronk, A.,

1416 Van Houdt, W., Van Gorp, J., Taylor-Weiner, A., Kester, L., et al. (2015). Prospective 1417 derivation of a living organoid biobank of colorectal cancer patients. Cell 161, 9331418945.

1419 WHO (2020). WHO Classification of Tumours: Soft Tissue and Bone Tumours. 5th 1420 Edition.

1421 Yamazaki, S., Ohka, F., Hirano, M., Shiraki, Y., Motomura, K., Tanahashi, K., 
1422 Tsujiuchi, T., Motomura, A., Aoki, K., Shinjo, K., et al. (2021). Newly established

1423 patient-derived organoid model of intracranial meningioma. Neuro. Oncol. 23, 1936-

14241948.

1425 Yang, J., Ren, Z., Du, X., Hao, M., and Zhou, W. (2014). The role of mesenchymal

1426 stem/progenitor cells in sarcoma: update and dispute. Stem Cell Investig. 1, 18-18.

1427 Yohe, M.E., Gryder, B.E., Shern, J.F., Song, Y.K., Chou, H.C., Sindiri, S., Mendoza,

1428 A., Patidar, R., Zhang, X., Guha, R., et al. (2018). MEK inhibition induces MYOG and

1429 remodels super-enhancers in RAS-driven rhabdomyosarcoma. Sci Transl Med 10.

1430 Yonish-Rouach, E., Resnftzky, D., Lotem, J., Sachs, L., Kimchi, A., and Oren, M.

1431 (1991). Wild-type p53 induces apoptosis of myeloid leukaemic cells that is inhibited

1432 by interleukin-6. Nature 352, 345-347. 
A

Tumor sample

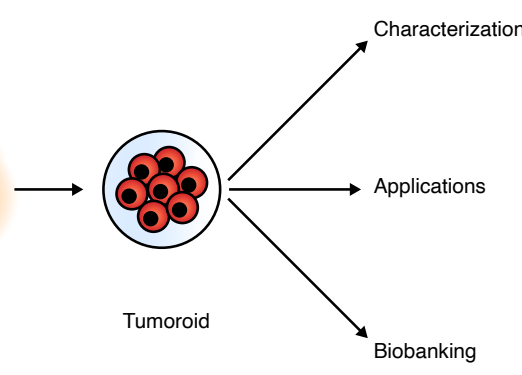

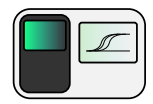

RT-qPCR
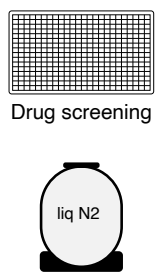

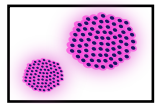

Histology

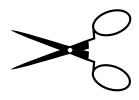

CRISPR/Cas9

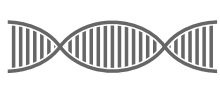

WGS \& RNA-seq

B

\begin{tabular}{|c|c|c|c|}
\hline & Primary site & Metastasis & \\
\hline Extremities & $\begin{array}{l}\text { (D) (1) (2**: } \\
\text { (D) (D) }\end{array}$ & & $\begin{array}{l}\text { embryonal histology } \\
\text { alveolar histology }\end{array}$ \\
\hline Abdomen & (2)* (1) & (1) & PAX3-FOXO1 \\
\hline Paratesticular & (D) (D) (D) & & $\begin{array}{l}\text { PAX7-FOXO1 } \\
\text { РАХ3-WWTR1 }\end{array}$ \\
\hline Lymph node & & (D) (2) (2) & D Upon diagnosis \\
\hline Bone marrow & & (D) (2) & $\begin{array}{ll}1 & \text { First relapse } \\
2 & \text { Second relapse }\end{array}$ \\
\hline Subcutaneous & & (2) (2) $)^{* *}$ & \\
\hline Cerebral & & (1) & \\
\hline
\end{tabular}

C
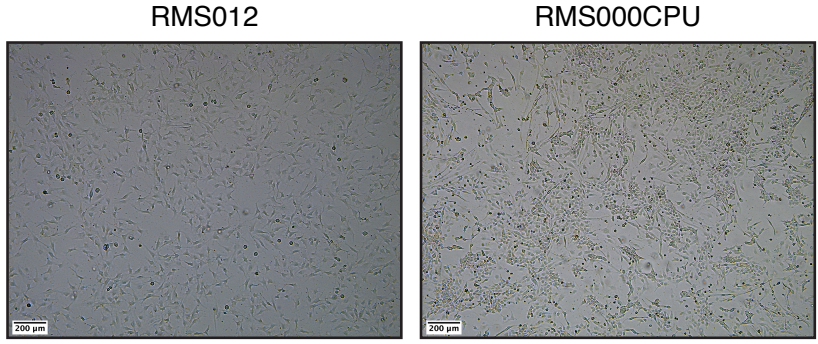

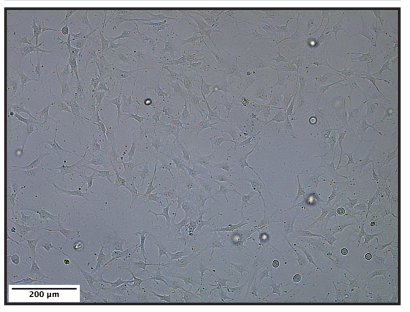

fusion-negative embryonal

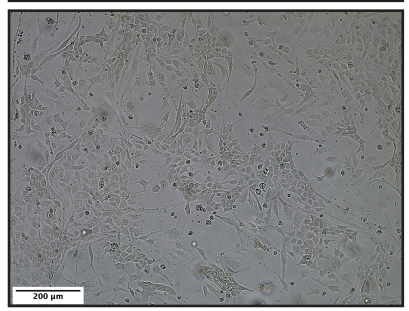

PAX3-FOXO1 alveolar 
Figure 2

A

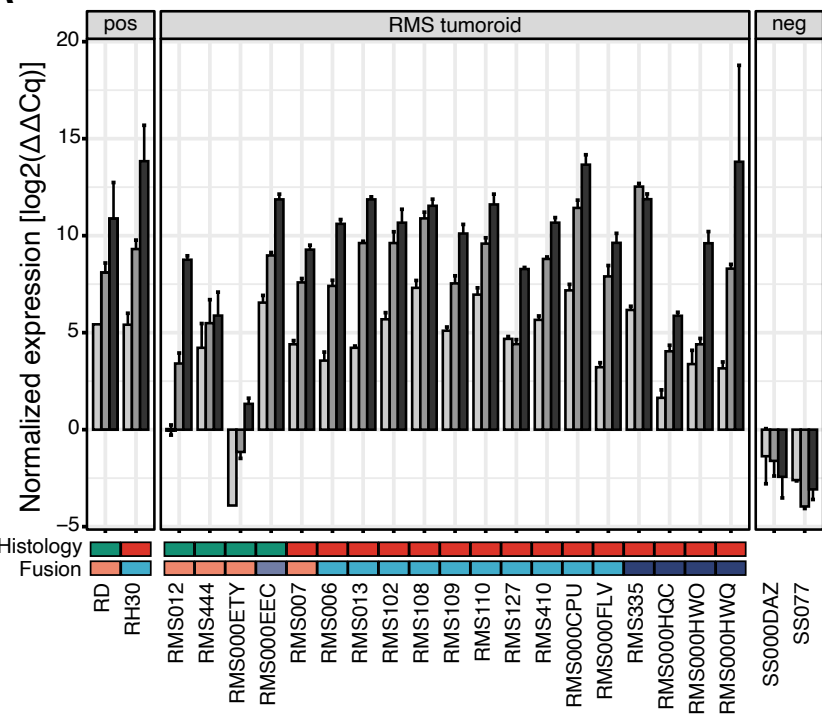

B

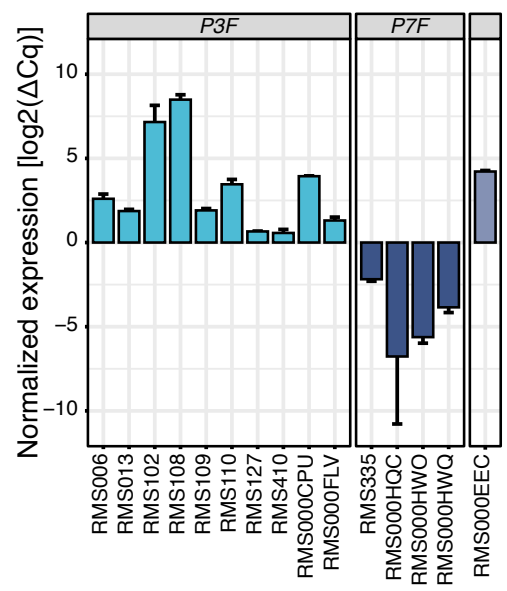

Fusion transcript

PAX3-FOXO1

PAX7-FOXO1

PAX3-WWTR1

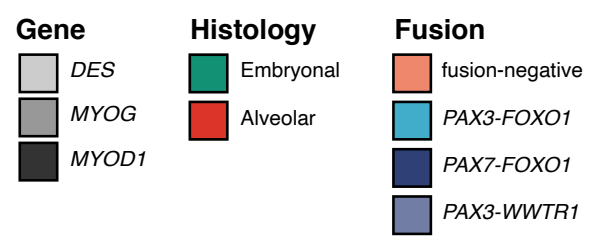

C

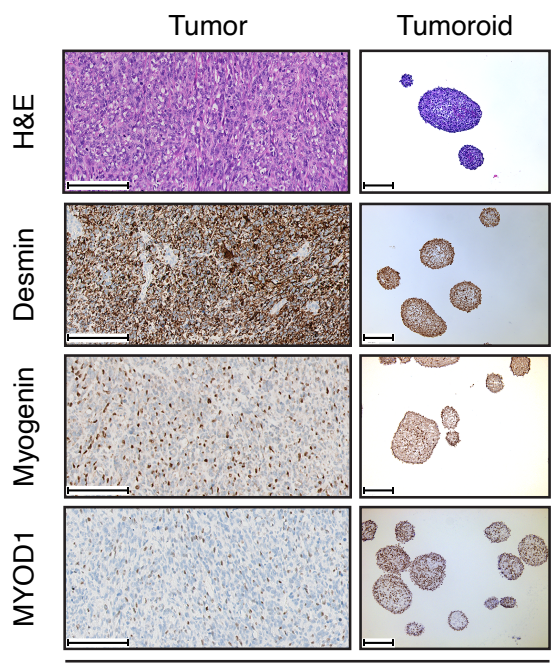

RMS012 (fusion-negative embryonal)

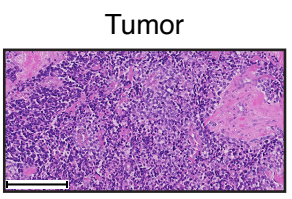

Tumoroid
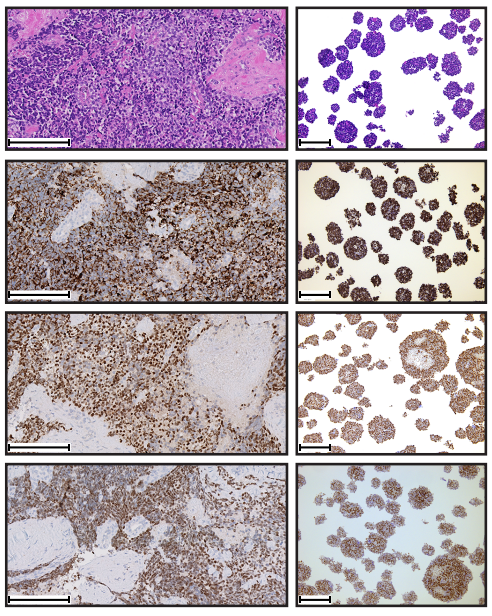

RMS102 (PAX3-FOXO1 alveolar)
Tumor
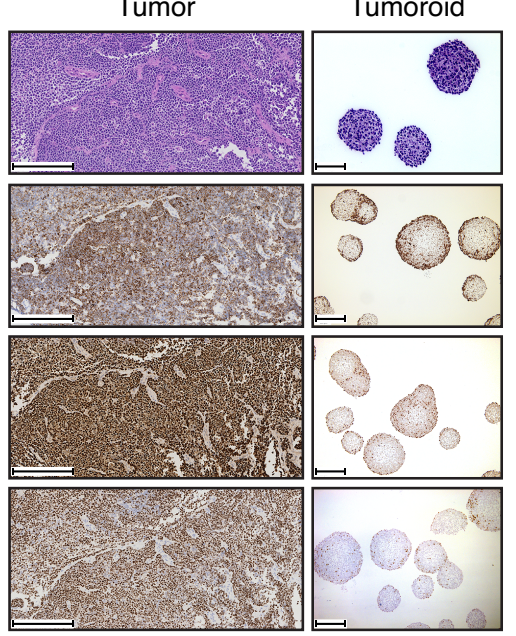

RMS000HQC (PAX7-FOXO1 alveolar) 
Figure 3

A

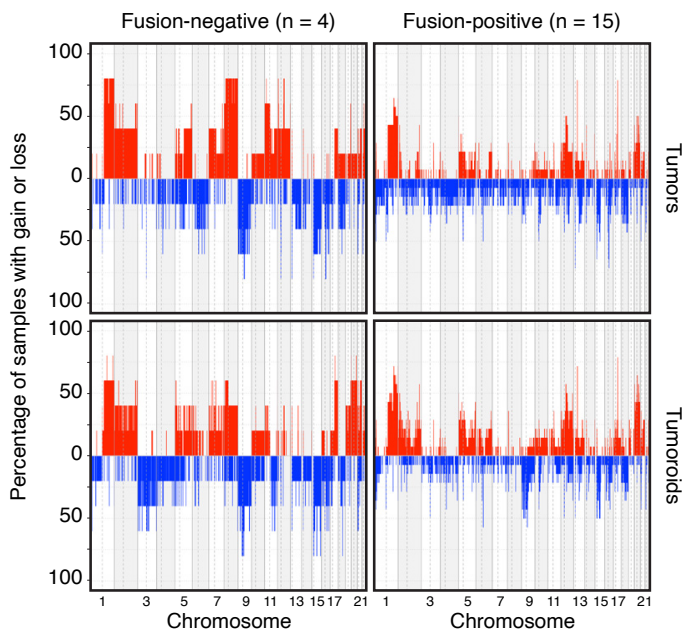

B

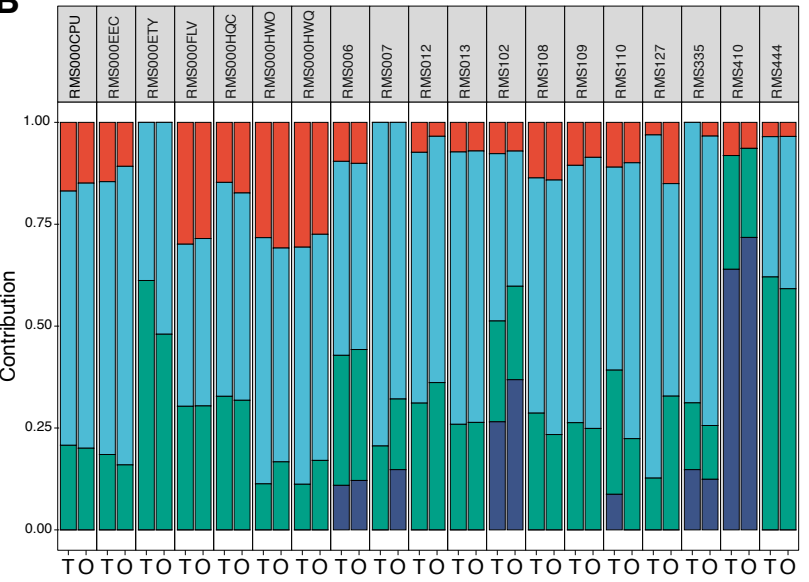

Signature

$\square$ SBS1 $\square$ SBS5 $\square$ SBS18 $\square$ TMZ

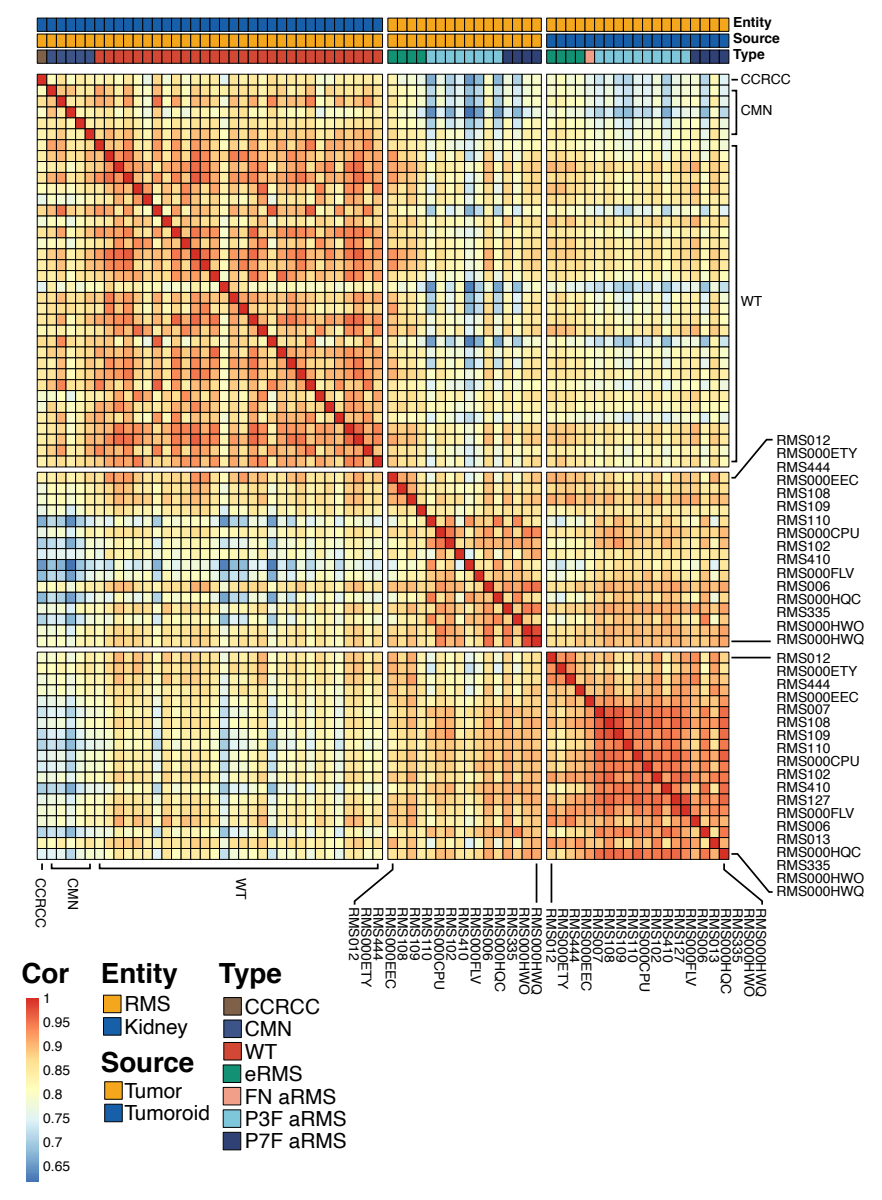

D

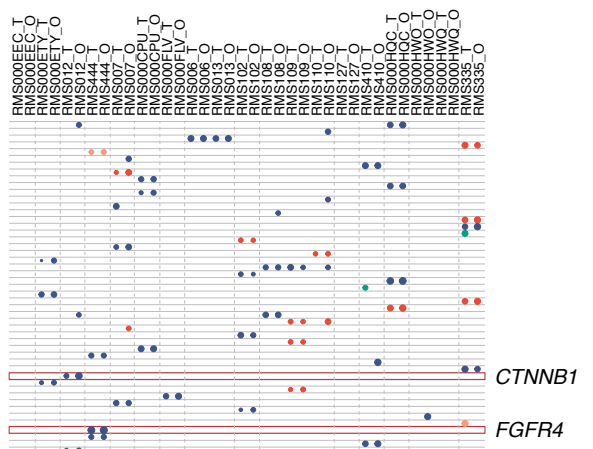

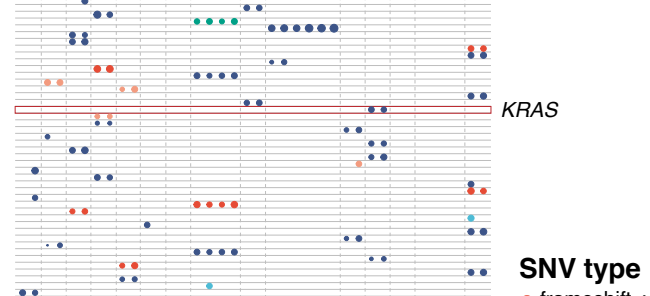

‥

- inframe_deletion

- inframe_insertion

- missense_variant

VAF

$\cdot 0.25$

$\bullet 0.90$

高

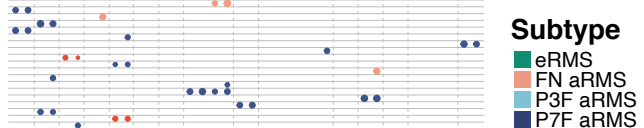


A
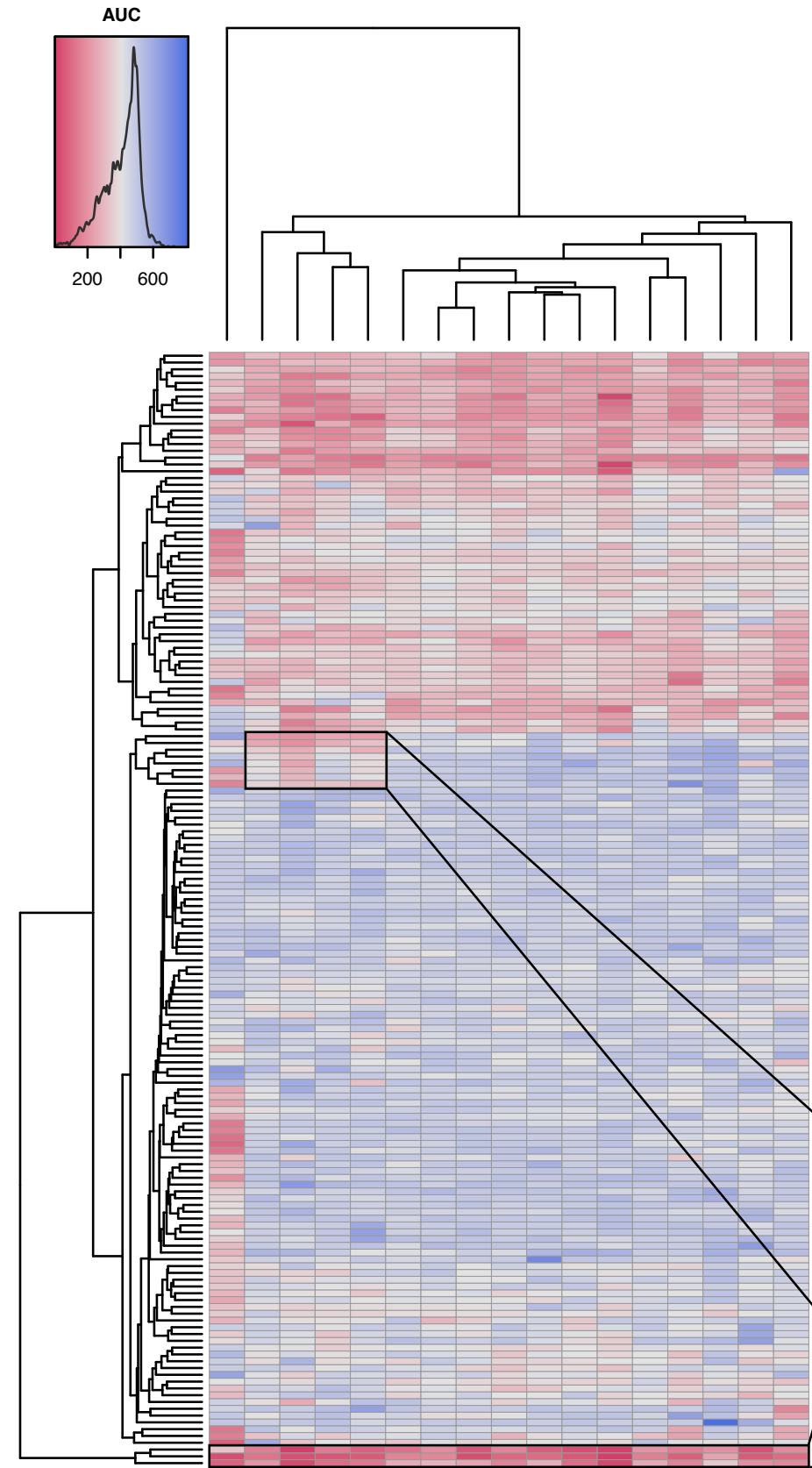

B

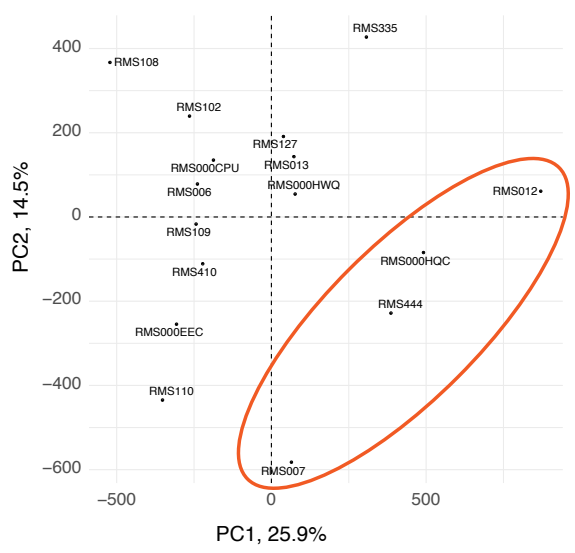

C

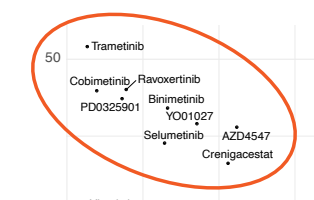

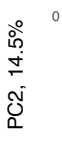

$-50$

(anblastine, Pacitiaxel

S63845 Irinotecan
Mitoxantrone

Alisertib
GSk10ican
GS100916

Etoposide
Talazoparib

$-50$

PC1, 25.9\%

Trametinib MEKi

Cobimetinib MEKi

PD0325901 MEKi

Binimetinib MEKi

$\begin{array}{ll}\text { YO01027 } & \text { Y-sec } \\ \text { Selumetinib } & \text { MEK }\end{array}$

Selumetinib MEKi

Crenigacestat $y$-secretase inhibitor

Ravoxertinib ERKi

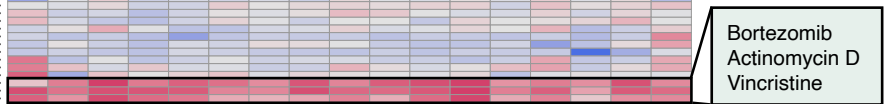

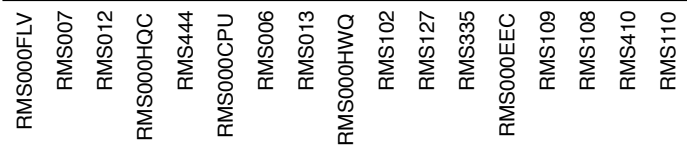

Actinomycin D

Vincristine 
A

TP53 wildtype

RMS tumoroid cells

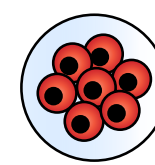

Transfection

TP53

sgRNA

B

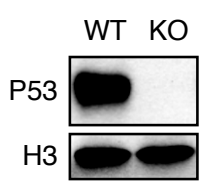

Polyclonal

population
TP53-KO

clones
Polyclonal TP53-KO

population

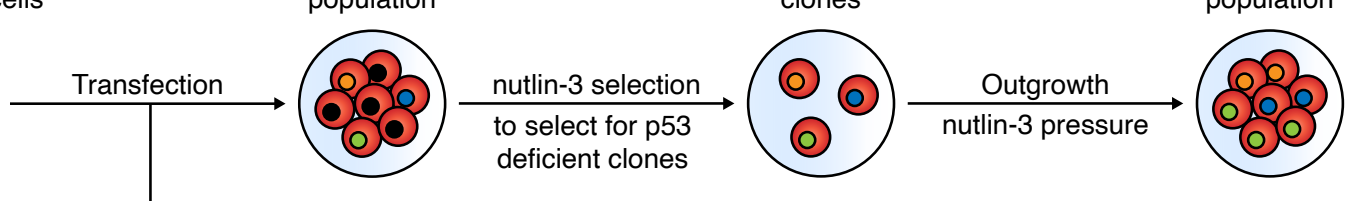

Cas9

C

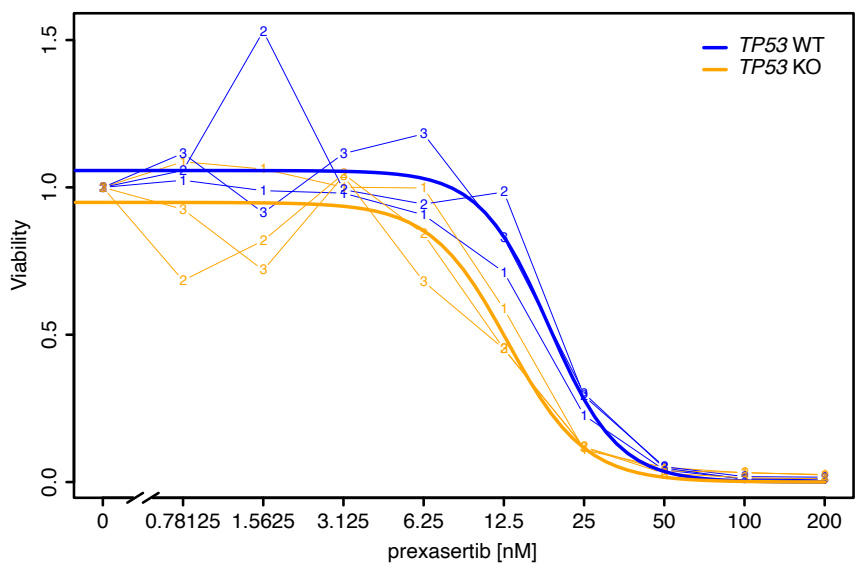




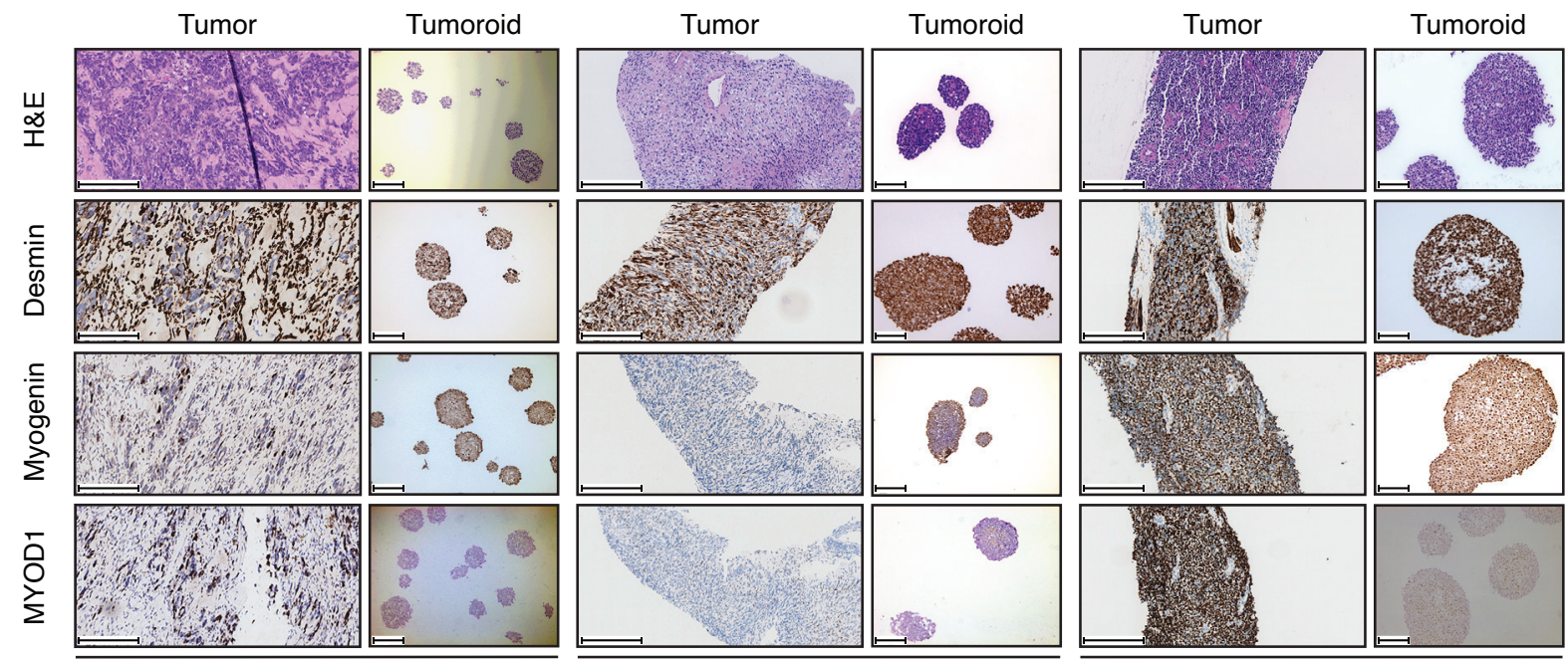

RMS007 (fusion-negative alveolar)

RMS000EEC (PAX3-WWTR1 embryonal) RMS000FLV (PAX3-FOXO1 alveolar)

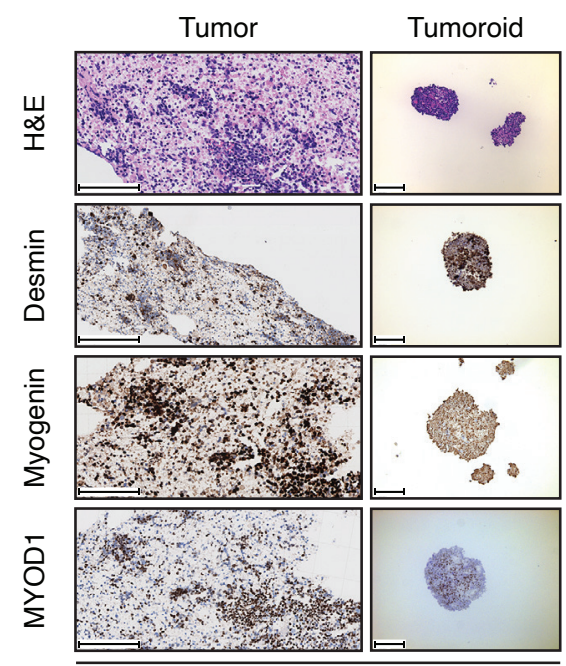

RMS000HWO (PAX7-FOXO1 alveolar)

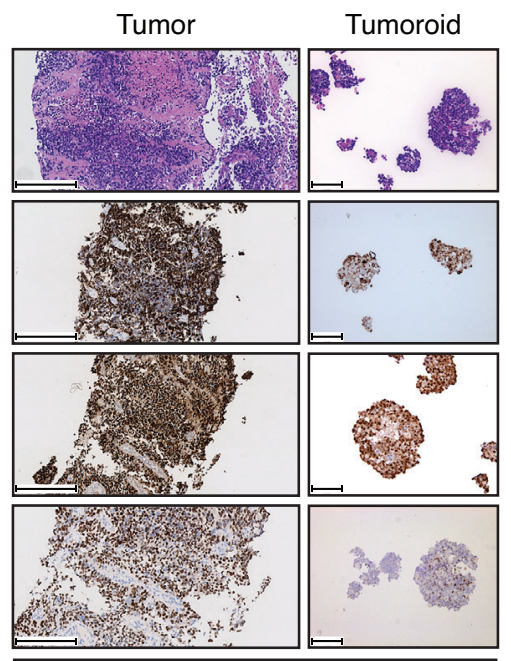

RMS000HWQ (PAX7-FOXO1 alveolar)

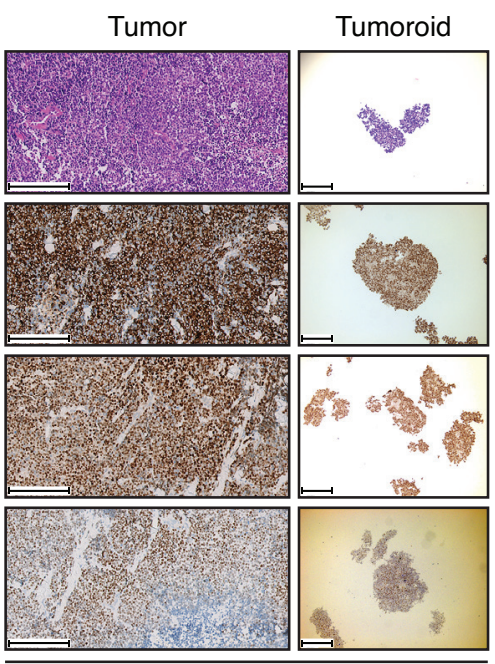

RMS335 (PAX7-FOXO1 alveolar) 


\section{Supplementary Figure 2}

A
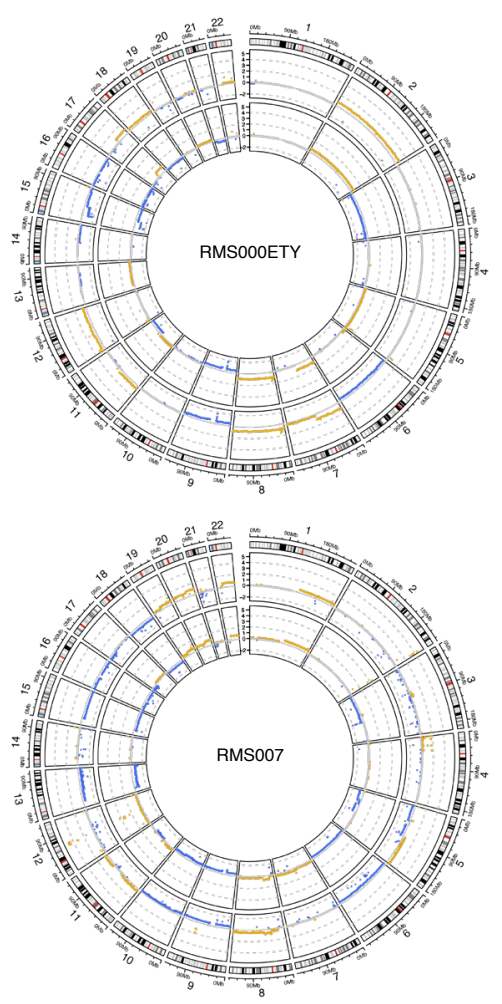

C

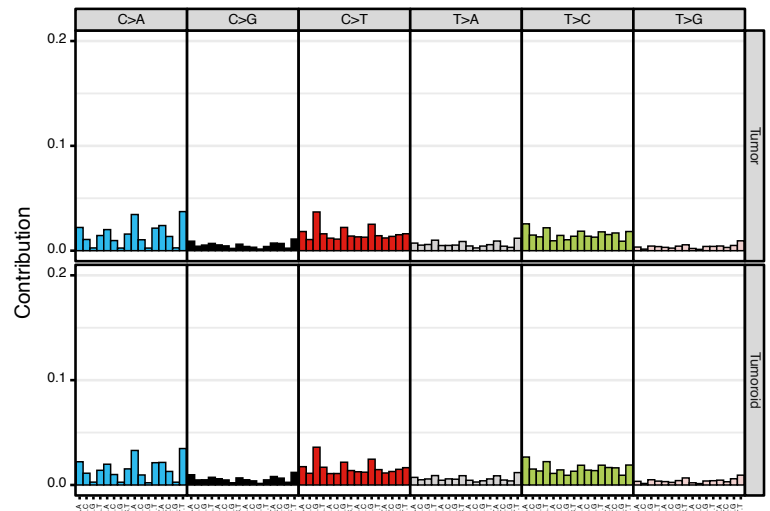

B

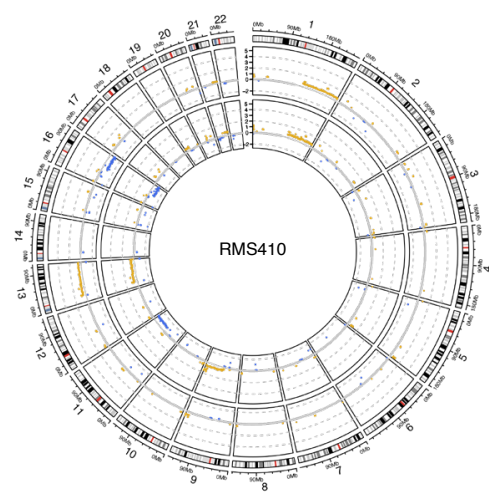

D
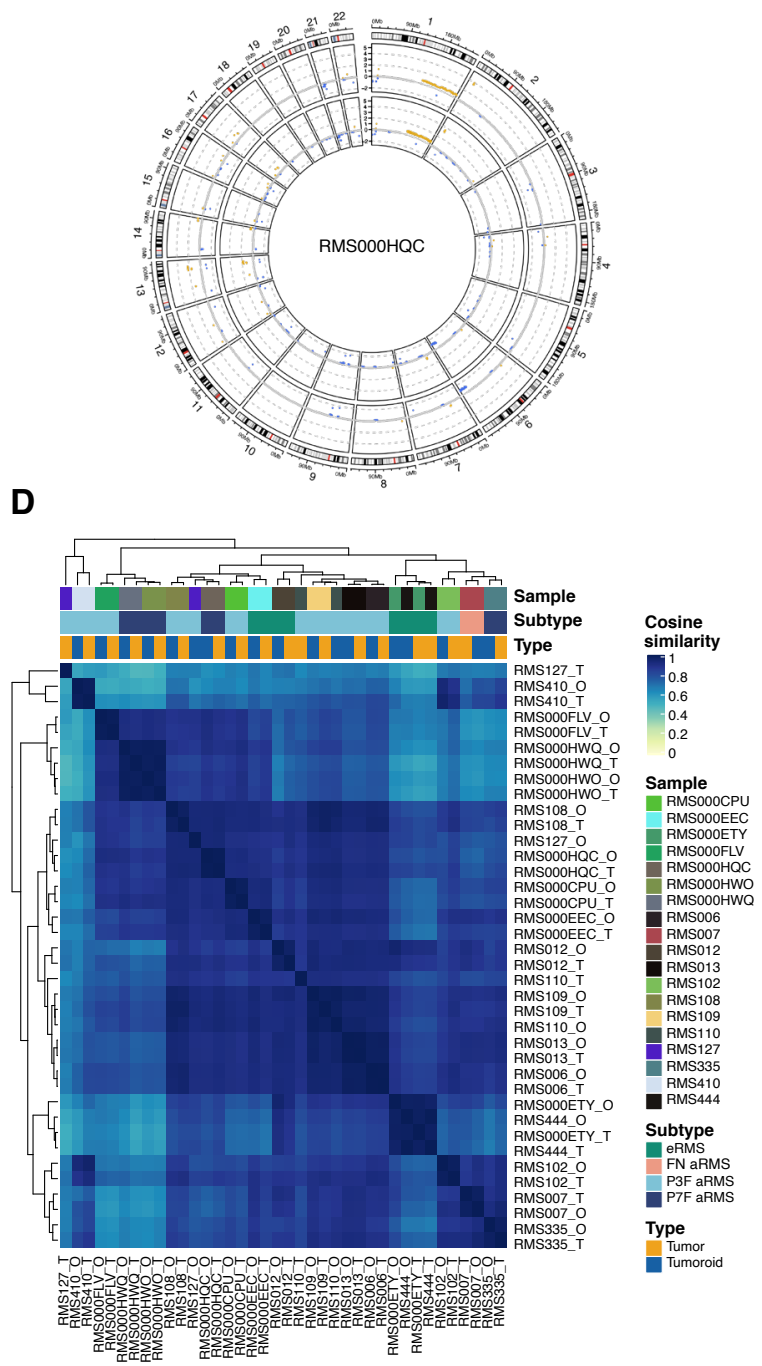


\section{Supplementary Figure 3}

A
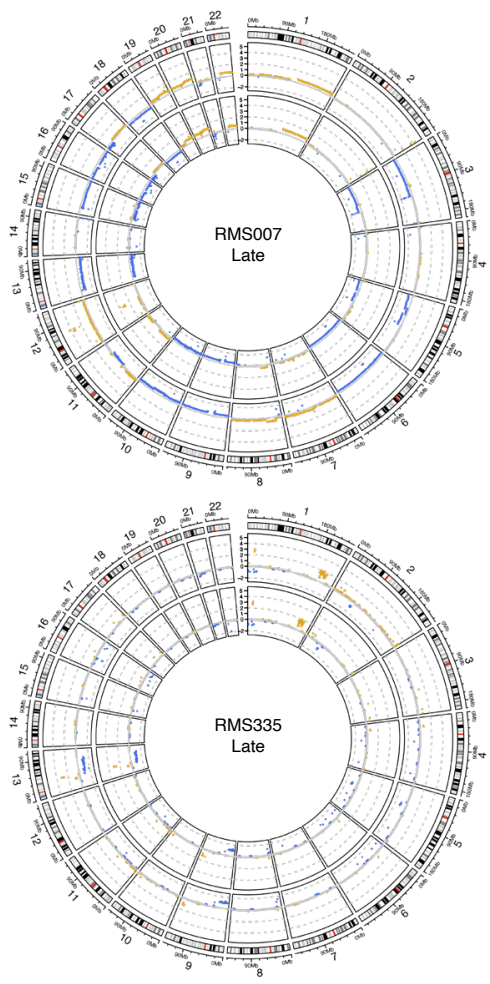

C

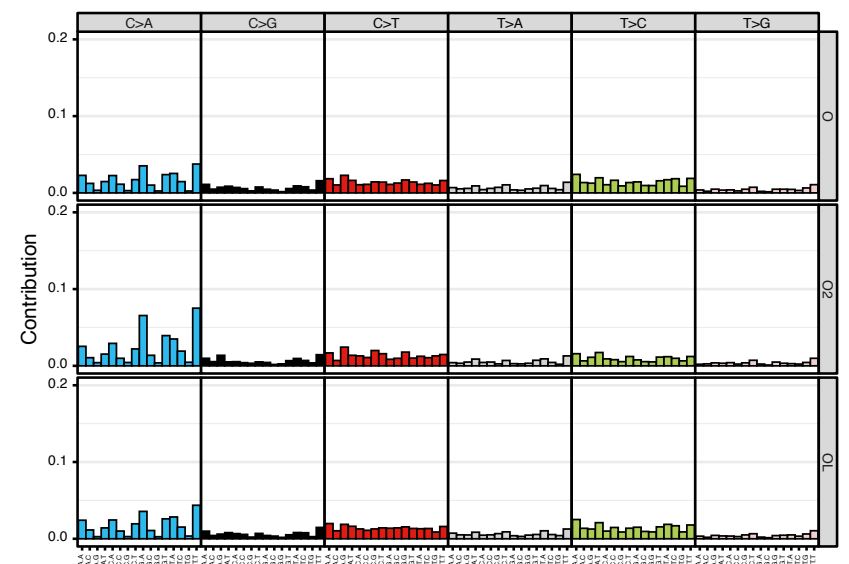

B
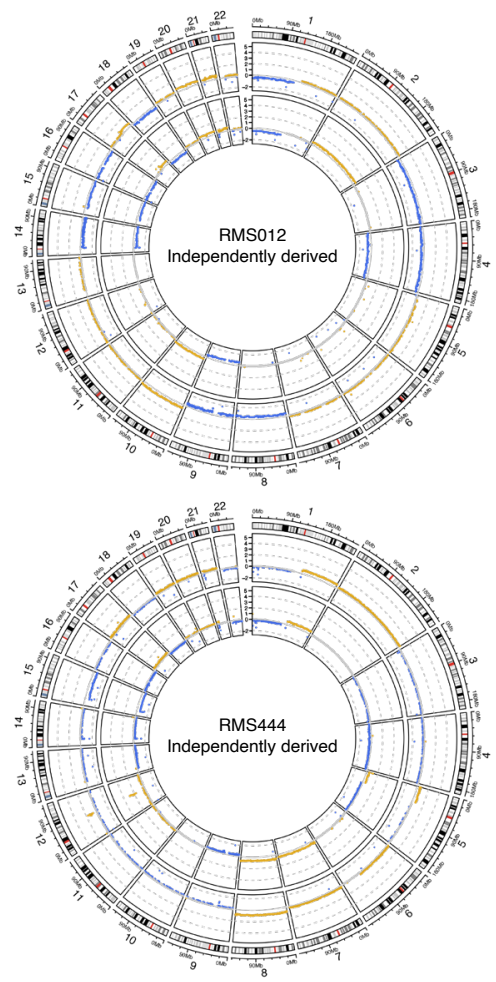

D

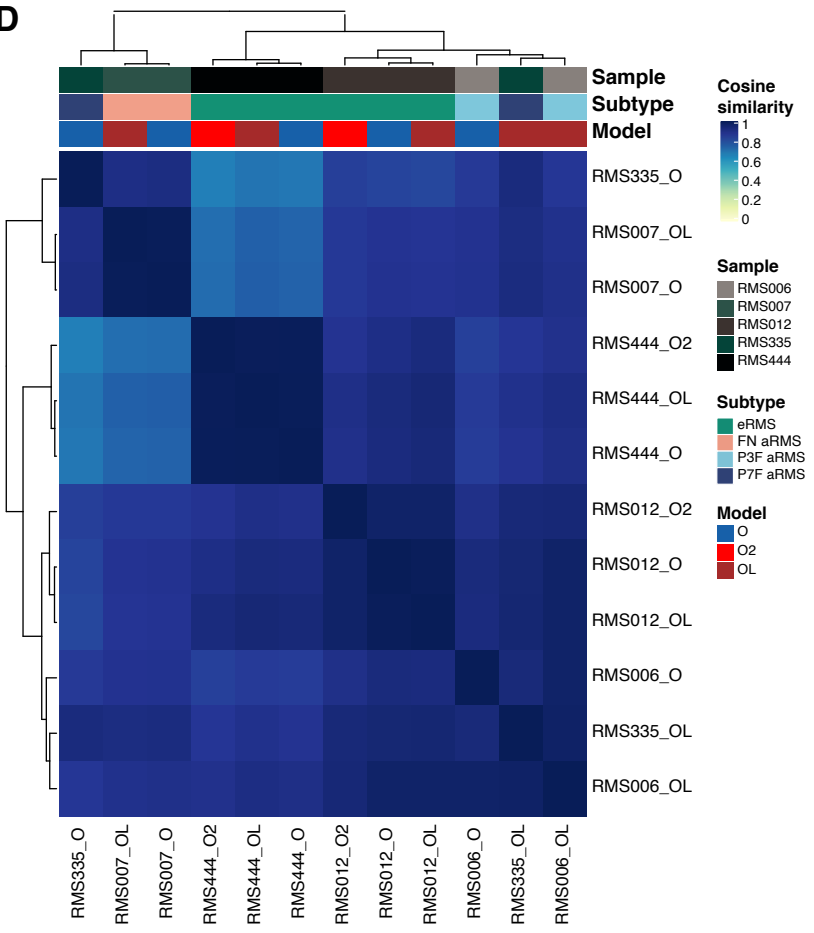




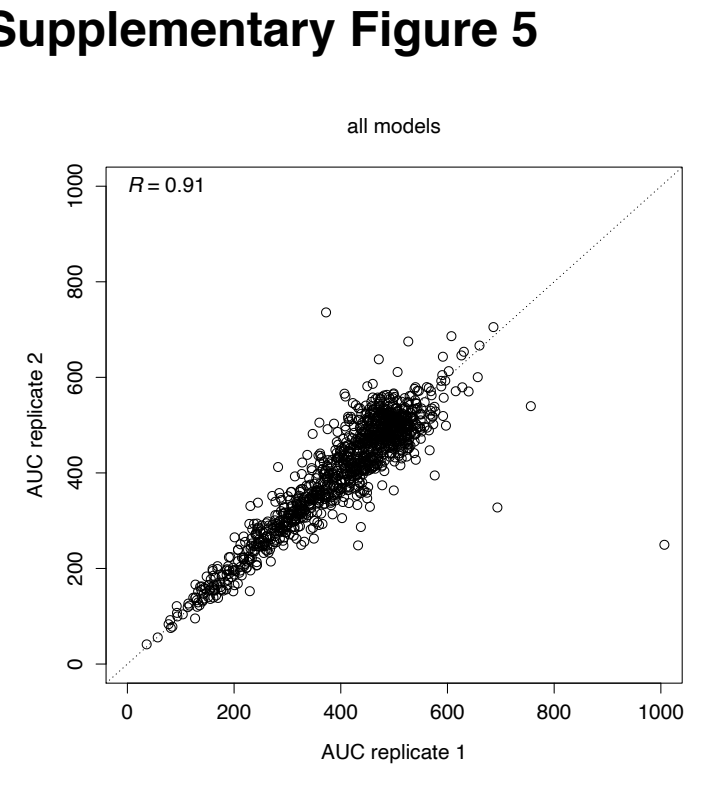

\section{Supplementary Figure 5}

AU replicate 1

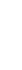
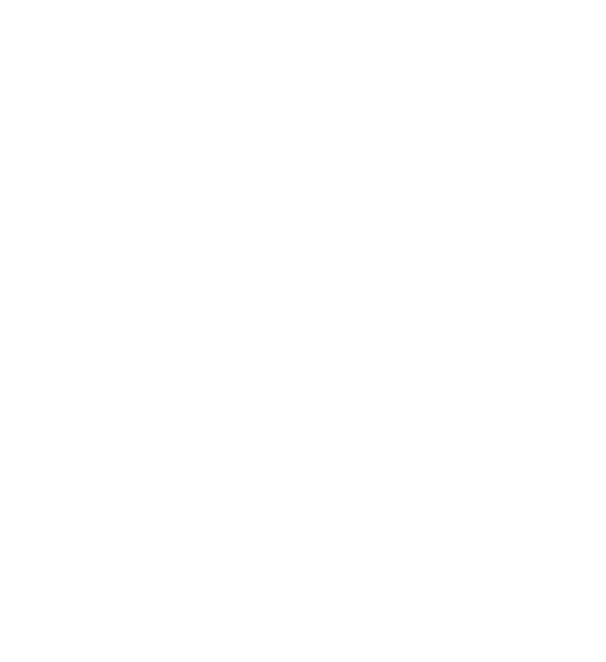